Aus dem k. k. hygienischen Institut des Prof. Dr. Gust. Kabrhel in Prag.

\title{
Der morphologische Metabolismus des lebenden Protoplasmas.
}

\author{
Von \\ Dr. Vladislav Rủžička, \\ Assistent an Institut. \\ Mit Tafel IV.
}

Eingegangen am 15. Februar 1906.

Inhaltsübersicht.

I. Ausgangspunkt der Analyse.

1. Cytoplasmatische Strukturen.

2. Kernstrukturen.

3. Das Centrosoma.

4. Die Spindel.

5. Kern und Cytoplasma.

6. Zelle und Grandsubstanz.

II. Analyse und Feststellung des Problems.

7. Definition des morphologischen Metabolismus des lebenden Protoplasmas.

8. Der morphologische Metabolismus als elementares Geschehen.

9. Die Voraussetzungen des Metabolismus.

10. Worin beruht das Problem des morphologischen Metabolismus?

III. Versuch einer Lösung.

11. Die Ursachen des morphologischen Metabolismus.

12. Schluß.

\section{Ausgangspunkt der Analyse.}

Vor allem habe ich die Tatsachen anzuführen, deren innerer Zusammenhang mich bestimmt hat, den nachfolgenden Versuch einer analytischen Bearbeitung zu unternehmen; ich halte sie im Interesse der tieferen Erkenntnis der lebenden Substanz fïr wünschenswert.

Es ist freilich nicht möglich, an diesem Orte alle jene Tatsachen, welche ich mit Bezug auf die unten zur Diskussion gebrachten Erscheinungen ror den Augen habe, aufzuzählen. Indem ich mir dieses 


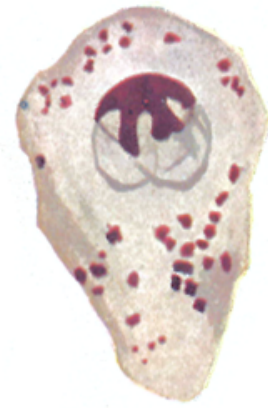

Fig. 1.

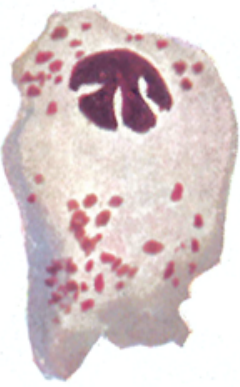

Fig. 2.

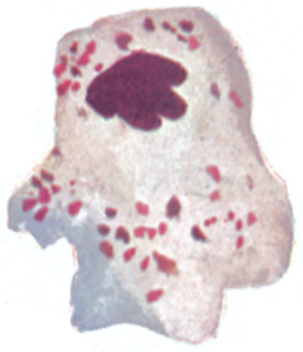

Fig. 3.

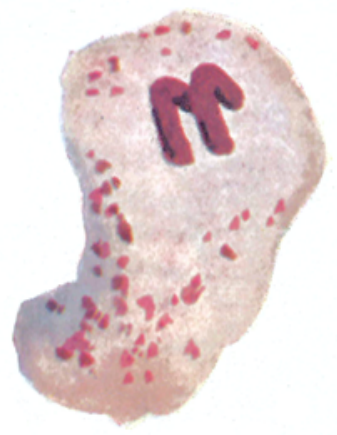

Fig. 4.

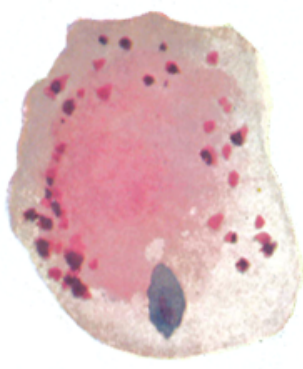

Fig. 5.

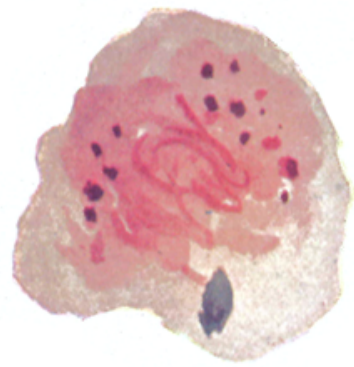

Fig. 6.

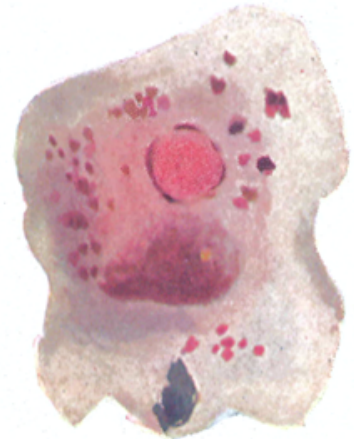

Fig. 7.
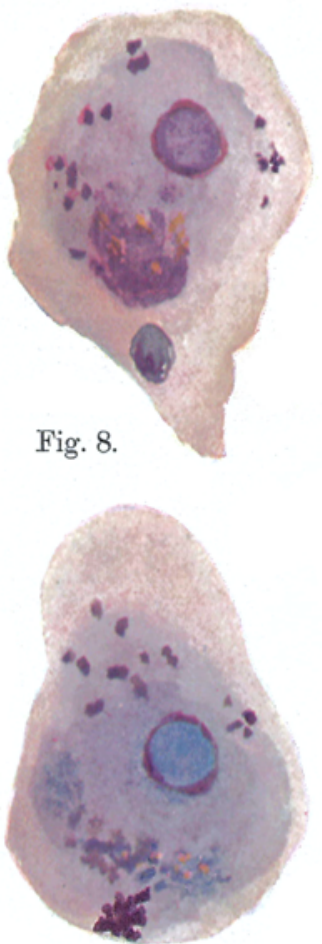

Fig. 9. 
für eine andre Gelegenheit anfspare, gestatte ich mir hier nur auf eine Reihe von typischen Fällen hinzuweisen, welche - wie ich hoffe - die Richtung, nach welcher hin meine Darlegungen sich bewegen werden, beleuchten und, meiner Meinung nach, auch genügen werden, um als Basis der nachstehenden Analyse zu dienen.

\section{Cytoplasmatische Strukturen.}

Auf Grund direkter Beobachtungen des lebenden Protoplasmas habe ich1) die Angabe gemacht, daß die einzelnen morphologischen Elemente des Cytoplasmas, als Granula und Fäden, und auch die aus denselben kombinierten (netzförmigen, schaumigen) Strukturen bei bestimmten Objekten (Bakterien, Lencocyten) als nicht stabile Gebilde erscheinen, indem sie vor den Augen des Beobachters untergehen und von neuem entstehen können.

Analoge Beobachtungen sind nun zwar auch bereits fruher von andern Autoren, z. B. Heitzmanx 2 ), Fromanx ${ }^{3}$, His ${ }^{4}$, Verwors ${ }^{5}$ ) u. a. an verschiedenen Objekten gemacht worden, es ist jedoch der Beweis nicht erbracht worden, inwieweit sich diese Erscheinungen am lebenden Protoplasma abspielen. Die betreffenden Elemente gehen nämlich in einer homogenen, mikroskopisch strukturlosen Grundsubstanz unter und aus derselben wiederum hervor. HeirzMaxy und eine Reihe andrer sprach jedoch diese Substanz, welche mit der in den Maschen der Schanmstruktur von fixierten Präparaten enthaltenen identisch ist, als tot, nicht lebend an. Max Schultze, Köldiker und ApÁthy haben sodann bezüglich der nicht stabilen Strukturgebilde die Meinung ausgesprochen, daß es sich (die Beobachtıngen bezogen sich hauptsächlich auf Granula) um periodisch entstehende und vergehende Stoffwechselprodukte handle.

Erst mit Hilfe meiner ${ }^{1}$ ) Methode der vital-letalen Tinktion wurde eine Lösung dieser Frage angebahnt und damit auch eine Analyse der fraglichen Erscheinung ermöglicht.

1) Rủžıčka, Zur Frage der Färbbarkeit der lebenden Substanz. Zeitschr. f. allg. Physiol. IV. 1904. - Über tinctorielle Differenzen zwischen lebendem und abgestorbenem Protoplasma. PfuÜGers Archiv. 107. 1905.

2- Heitzmans, Untersuchungen iiber das Protoplasma. Wiener akadem. Sitzungsber. 1873.

3. Fromann, Zur Lehre von der Struktur der Zellen. Jen. Zeitschr. 1875.

4) His, Studien am Salmonidenkeim. Abh. d. Kgl. Sïchs. Ges. d. Wiss. $1898,1899$.

5) Verworn, Die physiologische Bedeutung des Zellkerns. Pflügers Archiv. 51. 1892. 
Diese Methode beruht, wie ich kurz rekapitulieren will, anf dem Umstande, daß das lebende Protoplasma das Methylenblau, das tote aber das Neutralrot zu deren Leucoprodukten reduziert.

Benutzt man also diese Farbstoffe, deren Molekulargröße fast gleich ist, in einer äquimolekularen Mischung, so speichert das lebende Protoplasma sichtbar blo $B$ das Neutralrot, das tote jedoch nur das Methylenblau auf.

Die Färbung bezieht sich in vivo auf die Differenzierungen des Cytoplasmas, kann jedoch auch den Kern treffen. Sobald die Zelle abzusterben beginnt, beginnen die Cytoplasmadifferenzierungen und der Kern sich mit dem Methylenblau zu färben. Diese Tatsache wurde von mir an einer großen Reihe von Objekten festgestellt und habe ich keine Abweichnng von dieser Regel zu konstatieren rermocht.

Durch Anwendung dieser Methode kann also mit Sicherheit festgestellt werden, ob eine bestimmte gefärbte Differenzierung des Cytoplasmas lebt oder nicht - freilich aber nur im Falle einer positiven Färbung.

Denn ans meinen Beobachtungen geht auch hervor, daß sich die Protoplasmadifferenzierungen intravital weder alle auf einmal färben, noch daß sie stets gefärbt bleiben müssen, solange der Farbstoff einwirkt und daß sie sich schließlich tiberhaupt nicht tingieren mïssen. Das morphologische Bild wird nämlich durch chemische Erscheinungen kompliziert, welche zu einer alternierenden Oxydation und Reduktion des Farbstoffes führen. Ja, die Oxydation und Reduktion scheinen im lebenden Protoplasma stetig und zwar mit wechselndem Erfolge, beim Werke zu sein, da man oft beobachten kann, daß zwar die Färbung persistiert, jedoch an Intensität wechselt.

Doch muB, selbst wenn es zu einer vollständigen Reduktion des Farbstoffes zu dessen Leucoprodukt an irgendwelchem morphologischen Elemente des Protoplasmas kommt, das letztere dadurch nicht unsichtbar werden; davon haben mich viele meiner Beobachtungen überzeugt. Ebenso wie man im ungefärbten Protoplasma eventuell den Kern sehen kann, kann man auch die entfärbten Granula und Fädchen in demselben wahrnehmen und lägen sie selbst an der Grenze des Auflösungsvermögens des Mikroskops. Am besten konnte ich mich davon an den Strukturen lebender Bakterien überzeugen.

Kommt aber im Protoplasma irgend ein differenziertes Element zum Verschwinden, so kann dieses auch ohne Benutzung der vitalen Färbung gut verfolgt werden und zwar auf Grund der Änderung des 
Lichtbrechangsindexes. Um so mehr springt aber freilich ein derartiger Vorgang in die Augen, sobald man von der vitalen Tinktion Gebrauch macht.

Ich halte es für notwendig, diesen Umstand mit einigem Nachdruck hervorzuheben.

Bürschlı ${ }^{1}$ ) hegte nämlich seinerzeit die Meinung, daß die U'nsichtbarkeit von geformten Elementen im Protoplasma überhaupt durch den Umstand erklärt werden kann, daß dieselben den Liehtbrechungsindex ihrer Umgebung angenommen haben. Mit dieser Deutung hat sich offenbar auch Flemming ${ }^{2}$ ) bei Gelegenheit einer Besprechung der Unsichtbarkeit lebender Kernstrukturen identifiziert.

Einer derartigen Auffassung sind freilich schwer Einwendungen entgegenzustellen; es muß jedoch erwogen werden, daß die von derselben vorausgesetzte physikalische Änderung doch nur wieder durch eine physikalische Änderung des Substrates bewirkt werden konnte, infolge welcher sich das letztere mit Bezug anf das Beugungsvermögen der Lichtstrahlen jener Substanz, in welcher dasselbe verschwand, gleichstellt. Das Granulum, welches unsichtbar geworden ist, nahm also die optischen Eigenschaften der Substanz, in welcher dasselbe verschwunden war, an. Dabei kommt es offenbar auch zu chemischen Umwandlungen.

Davon kann man sich wiederum durch Auwendung der vitalen Färbung überzeugen.

Die Färbung, welche durch dieselbe an den geformten Elementen des Protoplasmas erzielt wird, ist nämlich distinkt, scharf begrenzt. Sobald jedoch ein derartig tingiertes Element zu verschwinden beginnt, wird seine Begrenzung unbestimmt, es hat das Ansehen, als wenn der Farbstoff in die Umgebung diffundieren würde (denselben Eindruck macht der Schwund des ungefärbten Granulums), zugleich blaßt er ab. Schließlich geht mit dem Schwunde des früher scharf begrenzten Elementes auch die Färbung völlig ein oder es verbleibt an der Stelle, welche jenes Element früher eingenommen hatte, eine blässere Färbung noch einige Zeit; doch besitzt diese Tinktion keine bestimmte Abgrenzung nach außen, kurz der ganze Vorgang ruft einen analogen Eindruck hervor, wie die Beobachtung eines im Wasser sich auflösenden Farbstoff körnchens. Der Schwund der Färbung bezeugt gleichfalls, daB die Substanz des dem Schwunde anheim-

1) Bütschl, Protozoa in Bronss Tierreich. 1889.

2: Flemming, Unsichtbarkeit lebendiger Kernstrukturen. Anat. Anz. 1892. 
gefallenen Elementes sich auch bezüglich ihrer Fürbbarkeit derjenigen Substanz, in welcher dasselbe untergegangen war, gleichstellt. Die letztere Substanz muß durch eine mächtige Reduktionsfähigkeit gegenuiber den basischen Farbstoffen ausgezeichnet sein, da sie stets ungefärbt bleibt - ansgenommen jene Fälle, in welchen ein gefärbtes Element in ihr verschwindet; doch ist wohl in diesen Fiillen allem Anscheine nach die Färbung noch auf die Rechnung der Substanz des Elementes zu setzen, welches sich in ihr auflöst.

Diese Beobachtungen sprechen entschieden für die Ansicht, daß es sich in den augefuibrten Fällen nicht um eine bloße Maskierung der Sichtbarkeit einer Differenzierung infolge Annahme eines Lichtbrechungsindexes, wie ihn die ubrige Protoplasmasubstanz besitzt, sondern im Gegenteil um einen tatsächlichen Untergang geformter Elemente, handelt.

Was sodann das Auftanchen neuer Strukturelemente betrifft, so bin ich in der Lage, in analogem Sinne zu deutende Beobachtungen anzuführen. Des Besonderen möchte ich hervorheben, daß bei den Bakterien die neu aufgetauchten Granula selbst eine metachromatische Färbung zu erkennen geben können, so daß in solchem Falle mit der morphologischen Differenzierung auch eine von dem früheren Zustande abweichende chemische Differenzierung vor sich gegangen. sein muß. Diese Fälle sind ron einer ganz besonderen Überzeugungskraft, indem sie für eine Neubildung der Granula sprechen.

Im ïbrigen kann man ja direkt das Hervorsprießen von Fäden aus den Granulis beobachten, durch welche sich die letzteren in gegenseitige Verbindung setzen - also die Neuentstehung von Strukturen. Derartige netzförmige Gebilde köunten wohl kaum für Nahrungsstoffe angesehen werden.

Nimmt man die Beobachtung mit Hilfe meiner vital-letalen Färbungsmethode vor, so gewinnt sie tibrigens noch ungemein an Überzeugungskraft.

Man kann daher als gesichert ansehen, daß die Strukturelemente des Cytoplasmas sich zu der zwischen denselben befindlichen formlosen Substanz umzuändern und aus der letzteren wiederum neu zu formieren vermögen und zwar in der Weise, daß man - wie ich an den Bakterien festgestellt habe -- eine (natürlich ganz willkürliche) Stufenleiter zusammenstellen kann, welche mit mikroskopisch strukturlosen Individuen beginnt, weiterhin Individuen mit rein körnigen, rein fädigen Strukturen faßt und mit kombiniert strukturierten $(\mathrm{d}$. h. mit kornfaden- und kornschaumigen Strukturen ausgestatteten Indi- 
viduen endigt, sowie weiterhin in der Weise, daß alle diese Strukturtypen auch in einem und demselben Individuum beliebig verbunden vorkommen können.

Diese Veränderlichkeit gelangt nicht nur an den allgemeinen Strukturen des Cytoplasmas, sondern auch an den speziellen Strukturen desselben zur Beobachtung.

Diesbezüglich mache ich auf meine Beobachtung des HerdexHaIxschen Mitoms ${ }^{1 j}$ eines Meerschweinchenleucocyten aufmerksam, die ich gleichfalls unter Benutzung meines vital-letalen Tinktionsverfahrens anzustellen in der Lage war. Die Radiensubstanz selbst färbte sich zwar nicht, um so besser aber die in dieselbe eingereihten Mitochondrien. Ich konnte jedoch beobachten, wie sich ein ungefürbter Radius direkt in ein Granulum umwandelte, welches sofort das Neutralrot aufnahm, weiterhin sah ich, wie ein andrer Radius sich in vier kleinere Granula verwandelte, die gleichfalls sofort den angeführten Farbstoff aufspeicherten.

Diese Beobachtung fubrt zur Anerkennung von Vorgängen, welche mit denjenigen analog sind, die nach der Beschreibung von BRANDT $^{2}$ ) bei der Ausbildung der Achsenstäbchen der Heliozoen in Aktion treten, wobei man im Auge behalten muß, daß diese Stäbchen nach einer Entdeckung von Schacdirn ${ }^{3}$ ) eventuell auch als Spindelfäden (also im Sinne eines Mitoms) zu fungieren imstande sind.

Schließlich möchte ich noch eine Beobachtung Verworss; in Erinnerung bringen. Dieselbe wurde an den Pseudopodien der Foraminifere Orbitolithes complanatus angestellt und zeigt ebenso wie die meinen deutlich, daB sich die beobachteten Wandlungen auf morphologische Elemente der lebenden Substanz beziehen. Nach den Angaben dieses Forschers treten an den Pseudopodien hier und da spindelförmige Verdickungen in Erscheinung, welche längs des Pseudopodiums gleiten und sich als strömende Körner darstellen, die sich jedoch da oder dort wieder zerteilen, um an einer andern Stelle neu zu entstehen und auf diese Weise zu beweisen, daß sie keine konstanten Gebilde sind. Solch ein spindelförmig ausgezogenes Korn kann sich sodann an irgendeiner Stelle rerdicken, in die Länge ziehen und zu einem neuen Pseudopodialast entwickeln.

1) RủžiěKa. Zeitschr. f. allg. Physiol. IV. 1904.

2) Zitiert bei Gurwitsch, Morphologie und Biologie der Zelle. 1904. S. 46.

3. Schaudinx. Centralkorn der Heliozoen. Verh. Zool. Ges. 1896.

4 Verworn, l. c. 
Durch diese Beobachtung wird somit gleichfalls festgestellt: 1) die Fähigkeit der Granula, sich zum strukturlosen Paraplasma umzuwandeln: 2) die Fähigkeit des Paraplasmas, sich zn differenzierten morphologischen Elementen umzuwandeln; 3) die Umwandlung einer Protoplasmadifferenzierung in ein Pseudopodinm, welche notwendig eine Differenzierung der ersteren in zwei Phasen (in strukturellem und chemisch-physikalischem Sinne voraussetzt.

\section{Kernstrukturen.}

Konschel' ${ }^{\text {1) }}$ hat über die Strukturen der Zellkerne der Raupen die nachfolgenden Mitteilungen gemacht. Man. kann einmal ein grobes, das andre Mal ein feines Netzwerk (z. B. bei der Raupe ron Pieris brassicae), oder eine feine Körnelung (bei Pieris rapae) zu Gesicht bekommen. In andern Fällen können die Fäden des Netzwerkes fehlen und die Knoten desselben als isolierte Granula auftreten. Es können jedoch auch alle angeführten architektonischen Elemente in einem und demselben Kerne gleichzeitig vorhanden sein. Einzelne Kerne enthalten nur wenige Körner, andre wiederum viele, manche können selbst $»$ vollkommen leer * scheinen, d. h. aus homogenem Plasma bestehen. Eine Reihe von Kernen zeigt schließlich teilweise oder insgesamt rein fädige Strukturen.

„Dieses Nebeneinandervorkommen scheinbar, leerer" und mit geformter Substanz geftillter Kerne ist merkwürdig genug", meinte KORSCHELT, ohne imstande zu sein dasselbe zu erklären, wiewohl er den Einwand, daß es sich um Artefacte handle, zurbickweist.

Auf Grund meiner im vorhergehenden Abschnitte zitierten Erfahrungen und Beobachtungen iiber die vitalen Wandlungen der Bakterienstrukturen, gewinnen jedoch diese merkwürdigen Erscheinungen eine klare, unzweideutige Beleuchtung.

Ich muß zugleich auch auf die Angabe von Fronsaxy ${ }^{2}$ ) aufmerksam machen, nach welcher dieser Forscher in den Kernen der Zwickelscheibe der Tulpe einen stetigen Wechsel der geformten Elemente ihres Inhaltes und zwar nach der Richtung hin beobachtet bat, daß jene Elemente untergingen und wieder neu auftauchten.

Weitere Belege für die Wandlungen der Kernstrukturen bringt

1) Konschelt. Beiträge zur Morphologie und Physiologie des Zellkerns. Zool. Jahrb. 4. 1891.

2. Fromaxx. Über Beschaffenheit und Lnwandlung der Membran, des Protoplasmas und des Kerns der Pflanzenzellen. Jen. Zeitschr. 1888. 
das Studium der mitotischen Vorgänge. Im Begiune der Mitose kommt es bekanntlich zur $\gg$ Konzentration des Chromatins auf den präformierten primären Faden «, einem Vorgange, bei welchem sich das Chromatin einesteils quantitativ vermehrt, andernteils jedoch dem Schwunde anheimfällt, indem die Seitenästchen, welche früher zur Bildung des gleichmäßigen Kernnetzes beigetragen haben, eingehen, ohne daß der Beweis geliefert worden wäre, daß es sich hierbei nur um eine einfache Wanderung des Chromatins von diesen Restchen auf den sprimären Faden handle. Denn in diesem Falle müßte das Liningerüst dieser Ästchen zurückbleiben, was jedoch nicht der Fall ist.

Mit Bezug auf die eben diskutierten Erscheinungen erscheinen die Befunde von Teluressiczkx ${ }^{1}$; von großer Wichtigkeit. Nach diesem Forscher besitzen die lebenden Kerne neben den Nucleolen mehrere unregelmäßig gestaltete, manchmal sehr feine Körperchen, welche auch Stäbchenform annebmen können, aus Chromatin bestehen und von Teluy essiczky als Nucleosomen bezeichnet werden. Diese Körperchen sind im Kerninhalte ganz frei gelegen, sie bilden nicht den Bestandteil einer präformierten, komplizierten Struktur. Weder die Nucleolen, noch die Nucleosomen müssen jedoch im Kerne vorhanden sein. Den einzigen konstanten und wichtigsten Bestandteil des Kerns bildet nach Tellyessiczky der "Kernsaft*. Derselbe ist aber vollkommen homogen.

Das Studium der angedeuteten Erscheinungen am lebenden Kerne ist mit bedeutenden Schwierigkeiten verbunden, welche sich noch dadurch vergrößern, daß die intravitale Färbung des Kerns nur manchmal gelingt und dann auch noch unter Bedingungen, welche wir bisher in keiner Weise festzustellen imstande waren. Bemerken muß ich jedoch, daß diejenigen ruhenden Kerne, welche ich bis jetzt im vital gefärbten Zustande zu beobachten Gelegenheit hatte, insgesamt ein völlig homogenes Aussehen zur Schau trugen.

Es erscheint auch mir stets wahrscheinlicher, daß alle übrigen Formationen der Kernsubstanzen, sofern sie überhaupt auf ritalen Verbältnissen beruhen, mit den Vorbereitungen zur Ausbildung der Chromosomen und mit der Bildung der letzteren selbst. nach abgelaufener Teilung aber mit dem Untergange dieser funktionellen Gebilde im Zusammenhange stehen.

1i Tellyessiczkr. Die Beschaffenheit der Kerne and ihr Verhältnis zur Hitose. Anat. Anz. Supplbd. 25. 1904. 
Bereits im Jahre 1897 habe ich die Meinung ausgesprochen 1;, daß diejenigen Bilder von Kernnetzwerken, welche zu jener Zeit als polare Anordnung. des Chromatins in ruhenden Kernen im Sinne von RABL gedeutet worden sind, wahrscheinlich als die ersten Anfänge der Konzentration * des Chromatins anzusehen sind, weil ich sie an Orten, an welchen ruhende Zellen vorausgesetzt werden mußten, außerordentlich selten gesehen habe. Diesen meinen Standpunkt sebe ich in letzter Zeit besonders durch die Arbeiten von TellyesNICZKY bestätigt.

Diesem Forscher gemäß breiten sich die Nucleosomen aus, entsenden $\ddot{A}$ ste, die daraus resultierenden Gebilde fallen der Vacuolisierung anheim, so daß schließlich von ihnen nichts mehr übrig bleibt und der Kern ein völlig homogenes Aussehen hat. Die Substanz der Nucleosomen mischt sich mit dem $\gg$ Kernsafte , aus welchem sich dann im Laufe der Vorbereitungen zur Teilung der "primäre Faden * herausbildet und zwar in der nachfolgenden Weise. "Zuerst glauben wir kleine Pünktchen zu sehen, dann fangen wir an zu ahnen, daß diese Pünktchen keine freistehenden Körperchen sind, sondern Bestandteile gewisser feiner Streifen; dann erscheint bald der feine Faden, welcher den ganzen Kern gleichmäßig ausfüllt. Darauf zeigt es sich, daß die zuerst sichtbar gewordenen kleinen Pünktchen den Winkelbrechungen des feinen Fadens entsprechen. Die Entwicklung des Kernfadens geht also von einem ganz diffusen Zustande aus, mit unendlich feinen Anfangsformationen.*

Ich erlaube mir, nochmals auf die prinzipielle Übereinstimmung dieser Angaben mit denjenigen von Konschelt und mir aufmerksam zu machen.

Da der Nucleolus zu den morphologischen Bestandteilen des Kerns gehört, so soll an dieser Stelle auch von demselben konstatiert werden, daß auch dieses Gebilde einer Umwandlung in andre morphologische Bestandteile der Zelle fähig ist.

Vor allem scheint dies bezïglich der Umwandlung des Nucleolus in die Chromosomen der Fall zu sein, auf welche hauptsächlich der Umstand hinweist, daß in den holoblastischen Eiern der Wirbeltiere und der Wirbellosen der Nucleolus das Hauptdepot des Chromatins bildet. Der Keimfleck verschwindet in zahlreichen Fällen im Beginne der Mitose oft spurlos. Deshalb wurde der Nucleolus früher

1. RủžıčKA, Učení o karyomitose v normè a pathologii. S. 4 d. Separatabdr. Vèstn. ¿̀. akademie. VI. 1897. 
des öftern für eine Anbäufung von Reservestoffen gehalten - ein Schicksal, das dieses Gebilde mit dem Kernchromatin teilte. Es ist jedoch selbstverständlich, daß einer solchen Anschaung nicht beigetreten werden kann. Verschiedene an den Nucleolen zur Beobachtung gelangende Erscheinungen, insbesondere aber die Art ihrer Beteiligung an der direkten Teilung der Zellen, zeigen klar, daß die Nucleolen aus lebendiger Substanz bestehen und also eine bei bestimmten Vorgängen in Funktion tretende Differenzierung des Protoplasmas darstellen.

Für diese Auffassung sprechen auch die Angaben von LACxoY ${ }^{1}$, welche die Beteiligung der Nucleolen der Drüsenzellen an den Secretionsvorgängen betreffen und zeigen, daß sich die Nucleolen hierbei entweder in zwei Teile oder zu einem feinen Staub zerteilen oder vacuolisieren oder schließlich im Kernsafte »auflösen".

In einzelnen Fiallen bildet sich aus dem Nucleolus die Spindel aus (STrasburgek), in andern verwandelt sich derselbe zum Cytoplasma 2).

Entstehung von Kernen aus Nucleolen wurde von R. HeRTwig an Thalassicolen konstatiert ${ }^{3}$.

\section{Das Centrosoma.}

Das Centrosoma muB ich gesondert besprechen, weil es als eine Zellorganelle von ganz besonderer Bedeutung, für die Zellteilung nämlich, angesehen wird.

Vor allem muß festgestellt werden, daß die Frage der Persistenz des Centrosomas in Zellen, in welchen die Wirksamkeit desselben während der Zellteilung über jeden Zweifel erhaben ist, in negativem Sinne beantwortet worden ist. CaRnoy und Lebrun 4) waren ebenso wie Fick ${ }^{5}$ ) außerstande in den Richtungsspindeln der Eizellen vieler Amphibien mit Sicherheit ein Centrosoma nachzuweisen. -

1) Launoy, Les phénomènes nucléaires de la secrétion. Arch. de sc. Zool. 1903.

2. Montgomery, Comparat. cytolog. studies with special regard to the morph. of the nucleus. Journ. of Morph. V. 1898. - 0. Hertwik, Morph. Jahrb. I. II. IV.

3) R. Hertwig, Zur Histologie der Radiolarien. Leipzig 1876.

4) Carnoy et Lebrun, La vesic. germin. et les glob. pol. chez les batraciens. La Cellule. 1897. 1899. 1900.

5) FIck, Über die Reifung und Befruchtung des Axolotl-Eies. Zeitschr. f. wiss. Zool. 1893. 
Dasselbe haben Sовотта ${ }^{\text {) }}$ bei der Maus, Jordax ${ }^{2}$ ) bei Diemyctylus, Boveri bei Ascaris vergeblich angestrebt. Die gleiche Erscheinung wurde bei Säugetieren in pathologischen Fällen konstatiert, wobei in den Zellen ganz unregelmäßige, mehrpolige Mitosen vorgefunden wurden, welche keine Spur eines Centrosomas aufwiesen.

Des weiteren hat sich ergeben, daß die sog. Quadrille des centres der Seeigeleier nicht, wie FoL gemeint hatte, sowohl vom mütterlichen als auch vom väterlichen Centrosoma abstammt, indem yon Boyeri, Wilsox, Reinke, Kostraxecki die Erkenntnis angebahnt worden ist, daß das Eicentrosoma zur Bildung der Furchungsspindel nicht mehr beiträgt, sondern im Gegenteil untergeht.

Wann und wie freilich das Centrosoma in den angedeuteten Fällen dem Schwunde anheimfällt, ist bis jetzt nicht konstatiert worden.

LiLlie aber hat mitgeteilt ${ }^{3}$ ), daß dieser Vorgang in dem Ei von Unio in der Weise vor sich geht, daß das Centrosoma sich samt der Sphäre in ein grobalveoläres Plasma umwandelt, welches einige Zeit hindurch von dem umgebenden Eiplasma abgesondert bleibt. Die Differenzen zwischen den beiden verwischen sich jedoch späterhin und das ganze Eiplasma erscheint später von einer kleinalveolären Schaumstruktur erfüllt, deren Alveolenwände aus winzigen Körnchen bestehen. Die Umwandlung des Centrosomas in das undifferenzierte Protoplasma wurde in diesem Falle Schritt für Schritt verfolgt.

Ich habe bis jetzt nur Fälle angeführt, welche bezeugen, daß das Centrosoma spurlos im Cytoplasma zu verschwinden vermag.

Es ist jedoch experimentell bewiesen, daB es aus dem letzteren auch neu entstehen kann. Die Überzeugungskraft der darauf bezüglichen Versuche ist ganz zweifellos. JACQUES LOEB ${ }^{4}$ ) hat es nämlich vermocht, durch Einlegen von unbefruchteten Seeigeleiern in bestimmte Lösungen von Zucker, Harnstoff oder Magnesiumchlorid dieselben zur parthenogenetischen Entwicklung zu bringen, welche vollkommen regulär bis zur Ausbildung ron Pluteus-Larven fortschreitet. Diese Tatsache wurde auch von andern Autoren bestätigt und wurde dasselbe Resultat auch durch Einwirkung andrer Faktoren z. B. der Hitze usw. erreicht.

1) SовоттA. Die Befruchtung und Furchung des Eies der Maus. Arch. f. mikr. Anat. $189 \tilde{\delta}$.

2. JoRDAn, Habits and development of the Newt. Journ. of Morphol. 1893.

3. Lillie, The Organisat. of the Egg of Unio etc. Journ. of Morphol. 1901.

4) Loes, On the nature of the princip of fertilisat. etc. Amer. Journ. of Physiol. 1899. 
Die Bedeutung dieser Entdeckung für die vorliegenden Erwägungen ist aus dem Umstande zu ersehen, da $B$ in den angefuhrten Fällen das Centrosoma unumgänglich aus dem undifferenzierten Protoplasma de novo entstehen mußte, da das reife Ei durch Austreibung der beiden Polocyten seines Centrosomas verlustig wird, das Furchungscentrosoma aber von dem Spermacentrum stammt. Das letztere war jedoch in den angeführten Fällen, in welchen unbefruchtete Eier vorlagen, nicht zugegen.

Es hat also als bewiesen zu gelten, daß das Protoplasma durch verschiedene künstliche Einflüsse zur Ausbildung von Differenzierungen gebracht werden kann, welche in demselben vorher nicht enthalten waren und daB auf solche Weise selbst Differenzierungen von der Bedeutung, wie sie dem Centrosoma beigelegt wird, entstehen können.

Mit den angeftihrten analogen Beobachtungen haben MEAD ${ }^{1)}$ und Reinke, von botanischen Objekten MotTIER ${ }^{2}$ ) veröffentlicht.

Wichtig sind die nachfolgenden Umstände. In den oben zitierten Fällen entstand das Centrosoma aus dem Cytoplasma.

Nach den Studien R. Her'rwigs ${ }^{3}$ ) tiber die sog. Reductionsteilung der Cysten von Actinosphaerium entsteht aber bei diesem Objekte das Centrosoma im Gegenteil aus dem Kerne und zwar aus der Kerngerüstsubstanz (dem Chromatin). Ein Teil des letzteren verdichtet sich, tritt aus dem Kerne heraus und bildet ein unregelmäßig alveoläres Centrosoma, dessen Substanz einesteils durch Reduktion, andernteils durch Verdichtung das typische Aussehen gewinnt.

Wie WassiLjEW ${ }^{4}$ ) angibt, entstammt das Centrosoma bei der ktinstlichen Parthenogenese durch Magnesiumeinwirkung wiederum aus der achromatischen Kernsubstanz.

NekrassofF ${ }^{5}$ ) zufolge entsteht schließlich das Furchungscentrosoma im Ei von Cymbulia Peronii durch gegenseitiges Zusammenwirken des Kerns und des Eiplasmas.

Vergleicht man endlich die Resnltate WassiLjews mit den von

1) Mead, Origin of the Egg-Centrosomes. Journ. of Morphol. 1897.

9) Motrier, Kernteilung in den Pollenmutterzellen einiger Dicotylen und Monocotylen. Pringsheims Jahrb. 1897.

3) R. Hertwig, Kernteilung usw. vom Actinosphaerium. Bayr. Akad. München. 1898.

4) WAssiluEw, Über künstliche Parthenogenese des Seeigeleies. Biolog. Centralbl. 1902.

5) Nekrassoff, Lntersuchungen über die Reifung und Befruchtung des Eies von Cymbulia P'ronii. Anat. Anz. 1903. 
WiLsox erzielten Ergebnissen, welcher die Centrosomenentstehung an kernlosen Fragmenten derselben Objekte (Echinodermeneier) beobachtet hat, so ergibt sich, daß das Centrosoma unter verschiedenen Verhältnissen selbst bei einer und derselben Zelle verschiedenen Teilen entstammen kann.

\section{Die Spindel.}

Über den Ursprung der Spindel wurde eine der hartnäckigsten Kontroversen geführt, welche die Geschichte der Histologie je verzeichnet hat. Hente vermögen wir bereits den richtigen Stand der Dinge zu uberblicken; derselbe stellt sich — sofern dies in den Kreis der vorliegenden Erwägungen fällt - folgend dar.

In den von Heidenhars beschriebenen Fällen erscheint das Postulat der direkten Umwandlung des Centrosomas zur Spindel nnumgänglich, da sich das erstere im Laufe des Wachstums der Spindel stetig verkleinert. Gemäß den Beobachtungen Boveris!) an den Eiern von Strongylocentrotus wird der größte Teil des bei diesem Objekte bedeutend großen Centrosomas abgeworfen, wodurch ein sog. reduziertes Centrosoma zustande kommt; der abgeworfene Teil aber wird zur Spindel. Sehr klar erscheint der analoge Vorgang im Ei von Rlynnchelmis nach der Schilderung von VEJporský und Mrázek 2). Bei diesem Objekte verwandelt sich das Centroplasma direkt in die Sphäre, die Sphäre umgekelrt wieder direkt in das Centroplasma.

Wie CaLKiss $^{3}$ ) zu zeigen in der Lage war, entsteht bei Noctiluca die Spindel durch eine Umwandlung der nicht zu Fäden differenzierten Sphärensubstanz. In den Orocyten der Polycladen treten nach den Befunden von GÉzard und Schockaer't ${ }^{\dagger}$ im undiffereuzierten Protoplasma zwischen den Dotterplättchen mit einem Male, ohne jeden Übergang, im Beginne vereinzelte, späterhin stets zahlreichere Fädchen auf, deren Entstehnng nur auf eine direkte Lmwandlung des Cytoplasmas zurïckgeführt werden kann.

$\mathrm{Zu}$ demselben Schlusse führen auch die an Toxopneustes-Eiern gewonnenen Ergebnisse WiLsoxs ${ }^{5}$ ). Das Protoplasma dieser Eier

1: Boveri, Zellenstudien. 1901.

2) VeJpovský und Mrazek, Veränderungen im Cytoplasma während der Reifung und Befruchtung der Rhynchelmis-Eier. Arch. t. mikr. Anat. 1903.

3. Calkixs, Mitosis in Yoctiluce milirris. Journ. of Morphol. 1899.

4 La Cellule. 1901.

5. Wizsox, On protoplasmic Structure in the Eggs of Echinodermes ete. Journ. of Morphol. $\mathbf{1 8 9 9 .}$ 
erscheint fein alveolär, die Alveolenwände bestehen aus feinsten Körnchen, welche auch am lebenden Objekte zu sehen sind. Die Spindelfäden entstehen daselbst nach den Mitteilungen WiLsoss durch longitudinale Anordnung eines Teils der erwähnten Körnchen, also durch Umwandlung des undifferenzierten Cytoplasmas.

In analoger Weise fand Boveri bei dem $\mathrm{Ei}$ ron Ascaris, daß auch in demselben die Spindel durch radiale Aneinanderreibung der cytoplasmatischen Strukturkörner zustande kommt und zwar in der Weise, daß die entstehenden Linien zu Anfang weniger zahlreich, kurz und undeutlich sind, aber desto schärfer hervortreten, je mehr sich die Centrosomen voneinander entfernen.

Mit Hinsicht auf die zuletzt zitierten Angaben muB ich neuerdings auf die auffallende Analogie in der eben beschriebenen Entstehungsweise der Spindel mit der Neubildung der Achsenstäbchen der Heliozoen, die ja zeitweise als Strahlen fungieren, sowie auf die Übereinstimmung dieser Erscheinungen mit meiner Beobachtung der Wandlungen im Mitome eines Leucocyten (Abschnitt 1) aufmerksam machen.

Zugunsten des Bestehens des umgekehrten Vorganges, also der Umwandlung der Spindel in das Cytoplasma, kann der Umstand angeführt werden, daß der Schwund der Spindel zur Zeit der Wanderung der Tochterchromosomengruppen zu den Polen vor sich geht. Da das Auseinanderrïcken der letzteren durch eine Kontraktion der Spindelfäden nicht bewirkt werden kann - es wird niemals eine Verdickung des kleineren Durchmessers der Fäden beobachtet - und die Verktirzung derselben mit dem allmählichen Schwunde derselben einhergeht, so muß wohl geschlossen werden, daß eben diese Verkürzung bereits eine Folge des Schwundes selbst ist. In sehr vielen Fällen kann (besonders in den Geschlechtszellen; von dem Untergange der Strahlen die Entstehung der sog. Nebenkerne abgeleitet werden, welche man heute als Cytoplasmadifferenzierungen ansieht. Obwohl dieselben mit den Sphären nicht verwechselt werden dürfen, so hat man trotzdem in ihnen bereits in mehreren Fällen Centrosomen aufgefunden. Im Beginne der Mitose, zur Zeit, wo die Centrosomen in Aktion treten sollen, fallen dann die Nebenkerne einem allmählichen Schwunde im Cytoplasma anheim.

Einen weiteren Fall ron besonderer Wichtigkeit stellt die Umwandlung der Kernsubstanz in die achromatische Figur dar. Ebenso wie wir in den oben zitierten Beispielen das Cytoplasma als einer Umwandlung in die Strahlen fähig erkanut haben, müssen wir dies 
auch von dem Caryoplasma anerkennen. Besonders bezieht sich das auf die Pflanzenzellen (Strasburger bei Fucus'), Swingle bei Stypocaulon $)^{2}$ ). Bei tierischen Objekten findet sich die nucleäre Spindel insonderheit bei Infusorien (Hertwig, Pritzner) und Rhizopoden (SchewidKofF), aber auch bei einigen Eiern (Boveri) vor. Trotzdem ist die Kernsubstanz der tierischen Zellen der obenerwähnten Umwandlung nicht in dem Maße fähig, wie diejenjge der Pflanzenzellen. Für die letzteren wurde in einigen Fällen der Ursprung der Spindel aus dem Nucleolus von Strasburger mehr als wahrscheinlich gemacht. O. HERTwiG ${ }^{3}$ ) stellte denselben auch für ein tierisches Objekt, das Ei von Asterias glacialis fest.

Eine noch größere Wichtigkeit kommt jedoch mit Bezug auf die vorliegend behandelten Erscheinungen denjenigen Fällen zu, in welchen (wie z. B. in den Spermatocyten von Ascaris nach Braler 4), bei den Copepoden nach RüCKERT) ${ }^{5}$ ) die Richtungsspindeln gemischten Ursprunges sind; weiterhin denjenigen Fällen kombinierter Spindeln, deren Mittelstück dem Kerne entstammt und selbst in die Kernmembran eingeschlossen erscheint, auf welche sich sodann cytoplasmatische Fadenkegel (z. B. bei dem Actinosphaerium nach Her'TwIG) anheften.

Die größte Bedeutung-nach dieser Richtung hin besitzen jedoch jene so auffallenden Fälle, in welchen bei Objekten, welche sonst bloß eine cytoplasmatische Spindel aufweisen, rein nucleäre Spindeln in Erscheinung treten, wie dies z. B. von WassiLjEW für Echinodermeneier bewiesen worden ist.

\section{Kern und Cytoplasma.}

Eine große Reihe von Erscheinungen weist auf Beziehungen zwischen dem Kern und dem Cytoplasma hin, welche in dieselbe Kategorie, wie die in den früheren Kapiteln besprochenen, einzureihen sind. Nur im Fluge kann ich andeuten, welche Erscheinungen ich da im Sinne habe.

1) Strasblrger, Kernteilung und Befruchtung bei Fucus. Jahrb. f. wiss. Bot. 1897.

2. Swivgle, Kern- und Zellteilung bei den Sphaeclanaceae. Jahrb. f. wiss. Bot. 1897.

3) O. Hentwig, Beiträge zur Kenntnis der Bildung, Befruchtung und Teilung des tierischen Eies. Norph. Jahrb. I. II. IY.

4) Braver, Zur Kenntnis der Spermatogenese von Ascaris megalorephala. Archiv f. mikr. Anat. 1893.

5) R 
Es sind vor allem zahlreiche Fälle des sog. cytoplasmatischen Chromatins, z. B. bei der Bildung der Dotterplättchen, in secernierenden Zellen, die sog. NissLschen Schollen der Nervenzellen usw.

Die grundlegende Beobachtung mit Bezug auf die angedeuteten Vorgänge hat im Jahre 1877 STRICKER $^{1)}$ angestellt, indem er an gewissen geformten Elementen des Froschblutes, welche er als nackte Kerne bezeichnete, teils die Ausbildung des Cytoplasmas - so daß aus dem urspriinglich nackten Kerne eine komplette Zelle entstand - teils den Schwund dieses cytoplasmatischen Saumes beobachtet hat - so daß sich eine vollständige Zelle in einen nackten Kern verwandelte. Außerdem konstatierte STRICKER an Froschleucocyten, daß die Kerne derselben entstehen, verschwinden und neuerdings auftauchen, wobei sich ihre Gestalt und Zahl verändert. Bei dem Schwunde des Kerns wird das Geriist desselben zu einem Bestandteil des Zellleibes, der Kern verwandelt sich somit in den Zellleib.

Diese Beobachtung Strickers wurde nicht bloß von seiten seiner Schule bestätigt, sondern anch von seiten andrer Forscher, von welchen ich nur Korscheli ${ }^{2}$;, Hayem ${ }^{3}$ ), Neumand ${ }^{4}$ ) und Griesbach ${ }^{5}$ ) anfuhre. Diese Forscher wichen nur in der Deutung jener Beobachtung von Stricker ab. Während Korschest jene Erscheinung durch bloßes Schwinden der Kernmembran erklären wollte, wobei die Substanz des Kerns erhalten bleiben sollte, haben die iibrigen Forscher sie als postmortal, nekrobiotisch, kurz als ein Zeichen des Zerfalles anfgefaßt.

Dem entgegen habe ich anzuführen: ich habe den Beweis geliefert $\left.^{6}\right)$, daß eine Zelle, welche den Schwund und die Neuentstehung des Kerns beobachten ließ, nach vollendeter Fixierung unter dem Deckglase (also bei ununterbrochener Beobachtung), denselben in einer Anzahl und Gestaltung erscheinen lassen kann, welche gegenüber der unmittelbar vor der Fixierung bestandenen verändert sein kann.

Außerdem bin ich imstande, nunmehr eine nene Beobachtung vorzulegen, welche ich vor zwei Jahren an einer aus einem Gras-

1) Strucken, Über die Entstehung des Kerns. Wiener akad. Sitzber. 1877.

2: 1. c.

; 3) Hayem, Rech. sur l'évolution des Hématies etc. Arch. de phys. norm. et path. 1879.

4) Teumaxx, Hämatologische Studien. Virchows Arch. 143. 1896.

5) Griesbach, Beitrüge zur Kenntnis des Blutes. Prü̈Gers Arch. j0. 1891.

6) Rùżička. Unters. über die ungefärbten Zellen des Blutes. Allg. Wiener med. Ztg. 39. 1894. 
aufgusse geziichteten Amöbe anzustellen in der Lage war und zwar unter der Benutzung meiner vital-letalen Tinktionsmethode (siehe Taf. IV).

Die Amöbe nahm das Neutralrot auf und zwar in der Weise, daß sich außer den Cytoplasmagranula auch der Kern gefärbt hat. Der Körper, den ich als Kern anspreche, besaß eine lappige Gestalt und zeigte im Laufe der Beobachtung Veränderungen, die in keiner andern Weise gedeutet werden können, als im Sinne der Schleifenbildung zum Zwecke der Zellteilung. Das ursprünglich gelappte, mit tief einschneidenden Buchten versehene Gebilde differenzierte sich im Laufe der Beobachtung in zwei deutlich abgesonderte, vollkommen scharf begrenzte und dunkel gefärbte Schleifen; der Körper der Amöbe führte verschiedene Bewegungen aus.

Nachdem aber die erwähnten Schleifen ausgebildet waren lösten sie sich mit einem Male im Körper der Amöbe auf und die Stelle, welche sie im Zellkörper früher eingenommen haben, erschien mit Neutralrot diffus homogen gefärbt. Die Färbung verlor sich gegen die Peripherie der Amöbe ohne jegliche Spur einer schärferen Begrenzung und erinnerte lebhaft an Lillies "nuclear area "1). Die gefärbte Area wies nicht die geringste Spur irgend einer Differenzierung auf.

Nachdem sie so einige Zeit bestehen geblieben, verdichtete sie sich schließlich zu einer Scholle, welche keine Struktur erkennen ließ und noch immer in einem mit Neutralrot diffus gefärbten Bezirke lag; aus ihr bildete sich schließlich ein typischer, ungeteilter, mit Nentralrot gefärbter Kern.

Der letztere, sowie auch die übrigen gefärbten Elemente, nahmen jedoch bald eine violette Färbung an - zum Zeichen, daß die Amöbe abzusterben begann. Tatsächlich stellte sie allmählich ihre Bewegungen ein, färbte sich samt dem Kerne immer klarer mit Methylenblau und verendete schließlich, nachdem sie fast genau die Kugelform angenommen (Taf. IV).

Ich lege auf die eben geschilderte Beobachtung um so mehr Gewicht, als sie mit Hilfe meiner Tinktionsmethode ausgefuhrt worden ist.

Auf diese Weise konnte nämlich gezeigt werden, daß die distinkte, auf die Kernschleifen beschränkte Färbung nach dem

1) Liluie. Different. without Cleavage in the Egg of the Annelid Chactopterus pergamentaceus. Areh. f. Entw.-Mech. 14. 1902. 
Schwunde derselben auf das Cytoplasma iibergegangen und gleichzeitig diffus geworden ist. Eine derartige diffuse Färbung kann aber sonst im Cytoplasma nie beobachtet werden und muß daber auf Rechnung der in demselben aufgehenden Substanz des schwindenden Kerns gesetzt werden, der ja gerade in diesem Falle vital gefairbt war. Die Färbung blieb auch nach dem Schwunde des Kerns rot (durch das Neutralrot bewirkt), es konnte sich somit um keine postmortale oder nekrobiotische Erscheinung handeln.

Des weiteren geschah in diesem Falle der Schwund des Kerns nicht in der von Konschelt vorausgesetzten Weise, d. h. durch einfachen Schwund der Kernmembran, denn die diffuse Area wies nach dem Untergange der Schleifen keine Struktur auf. Eine Kernmembran war in diesem Falle im Augenblick der Auflösung iaberhaupt nicht vorhanden.

Es kann somit geschlossen werden, daß unter bestimmten Umständen der Kern auch zur Zeit der größten Entfaltung seiner Funktion aufhören kann als morphologisches Element der Zelle zu existieren und daß sich derselbe aus dem undifferenzierten Protoplasma neuzubilden vermag.

DaB aber weiterhin die Kernsubstanz ohne gleichzeitige Mitwirkung eines Zellkörpers einer selbständigen Existenz fähig ist, zeigt der Beweis der Kernlosigkeit verbunden mit dem Nachweise des Aufbaues aus Kernsubstanzen, den ich $\left.{ }^{1}\right)$ für die Milzbrandbakterien und die roten Säugetierblutkörperchen, $\mathrm{MACALLCX}^{2}$ ) für die Cyanophycaeen und Beggiatoen geliefert haben.

\section{Zelle und Grundsubstanz.}

Wiewohl ich an dieser Stelle keine Definition des Begriffes der Grundsubstanz zu geben wünsche, so erlaube ich mir doch die Bemerkung zu machen, daß ich unter Grundsubstanz die Intercellularsubstanzen überhaupt verstehen will.

Ich bin mir dessen voll bewußt, daß die Tatsachen, auf deren Typus ich hier eingehen werde, zur Zeit noch immer Gegenstand der Diskussion sind und sich in der unten gegebenen Auffassung.

1) Rủžička, Über die biologische Bedeutung der fürbbaren Kürnchen des Bacterieninhaltes. Arch. f. Hyg. 47. 1903. - Weitere Untersuchungen über den Bau und die allgemeine biologische Natur der Bakterieu. Ibid. 51. 1904. Cytologische Studien über die roten Blutkürperchen. Arch. f. mikr. Anat. 67. 1905.

2) Mac Al.tur. On the cytology of non-nucleated Organismus. Unir. of Toronto Studies. 1900. 
keineswegs einer allgemeinen Anerkennung erfreuen; ich gestatte mir jedoch die Behauptung auszusprechen, daß dieser Nichtanerkennung auf keinen Fall eine unvoreingenommene Forschung zugrunde liegen kann, da man sich von den betreffenden Tatsachen eine positive Überzeugung zu verschaffen vermag. Vielleicht wird sich jedoch der Widerstand gegen die angedeutete Auffassung vermindern, wenn sich auf Grund der vorliegenden Darlegungen die erwähnten Erscheinungen nur als das Glied einer großen Kette andrer analoger Phänomene erweisen, deren großer Teil zu dem gesicherten Fond des Potentialbewnßtseins der Histologen gehört.

Die Wahl eines Beispiels zur Veranschaulichung der gegenseitigen Beziehungen zwischen den Zellen und der Grundsubstanz war mir nicht leicht. Verschiedene Umstände derselben treten in verschiedenen Geweben mit der größeren Überzeugungskraft auf.

Schließlich gab ich der Feststellung der betreffenden Tatsachen bei dem Sehnengewebe den Vorzug, da bei diesem Objekte auf Grund der fundamentalen Arbeiten von SpiNa ${ }^{1)}$ sowohl der Schwund als auch die Entstehung der Zellen klar beleuchtet erscheinen.

Wiewohl man, natuirlicherweise, die betreffenden Vorgänge bei diesem Objekte direkt zu beobachten nicht in der Lage ist, so geht die Tatsache der Umwandlung der Zellen in die Grundsubstanz schon aus einem Vergleiche der Struktur einer jungen und einer alten Sehne hervor.

Die Sehne sehr junger (neugeborener) Tiere wird aus Kolumnen prismatischer Zellen zusammengesetzt, welche eine Hülle und Ausläufer aus elastischer Substanz besitzen; diese Ausläufer sind in einer eben für die junge Sehne charakteristischen Weise sekundür und tertiär außerordentlich reich verzweigt und durchziehen als ein reichhaltiges Netzwerk die elastischen Fibrillenbuindel, welche zwischen den Zellen die Grundsubstanz darstellen. Die Quantität der Grundsubstanz ist also in der jungen Sehne nicht sehr groß.

Mit zunehmendem Alter verflachen sich aber die früher prismatischen Zellen und die Zellkolumnen erscheinen schließlich als elastische Bänder, in welchen die Kerne und die Grenzen der Gebilde, aus welchen sie entstanden sind, noch wahrgenommen werden können. Zngleich hat die Verzweigung der Ausläufer bedeutend abgenommen. Die Fibrillenbündel - die Grundsubstanz also - haben dagegen eine starke Vermehrung erfahren.

1) SpINa, Wiener mediz. Jahrbücher. 1873. 1877. 
Die Verflachung der Zellen, die Verminderung der Verzweigung der Ausläufer und umgekehrt das Anwachsen der Grundsubstanz weisen auf eine direkte genetische Beziehung zwischen den Zellen und deren Adnexen und der Intercellularsubstanz hin. Diese Beziehung wird um so deutlicher, wenn man erwägt, daß die Grundsubstanz aus elastischen Fibrillen besteht, ebenso wie die Ausläufer und die Hüllen der Sehnenzellen und wenn man sich erinnert, daß - wie O. Hertwig $\left.{ }^{1}\right)$ wahrscheinlich gemacht hat - die elastische Substanz durch direkte Umwandlung der Protoplasmaoberfläche gewisser Zellen entsteht.

Es liegt also auf der Hand, daß der auffallende Unterschied in der Zusammensetzung der jungen und der alten Sehne am besten von einer direkteu Umwandlung der Zellen in die Grundsubstanz abgeleitet werden kaun.

Daß man sich auch die erste Entstehung der Sehnengrundsubstanz in der gleichen Weise vorstellen muß, dafür sprechen die Angaben von STricker ${ }^{2}$. An der Sehne eines $6 \mathrm{~cm}$ langen Kaninchenembryos kann man nach STricker sehen, daß die Kolumnen prismatischer Zellen zwischen gleich prismatischen Zellen liegen, die jedoch keine Zellkolumnen mehr sind, sondern Fibrillenbündel. War aber diese Sehne - so lauten die Erwägungen Strickers - auf einem. noch jüngeren Stadium ans Zellkolumnen znsammengesetzt, so mußte sich ein Teil derselben zur Grundsubstanz umwandeln. Dieser Schlußfolgerung kann die Zustimmung sicherlich nicht rerwehrt werden. Bereits RoLLET ${ }^{3}$ ) schloß, daß die homogene Substanz, aus welcher die Fibrillen entstehen, durch Zusammenfließen umwandelter Zellen zustande kommt. In der Sehne müssen jedoch zwei Vorgänge vor sich gehen: zum Ersten entsteht die intercelluläre Substanz (die Fibrillenbündel) aus den Kolumnen zusammengeflosseuer Zellen, zweitens aber wächst sie (beim altern) infolge einer $»$ formativen * Tätigkeit der persistierenden Zellen, die an Substanz verlieren, während die Fibrillenbtindel anwachsen.

Was anderseits die Entstehung von Zellen aus der Grundsubstanz betrifft, so muß freilich zum $Z_{w}$ ecke ihrer Erkenntnis zum Studium der Sehnenentzündung gegriffen werden; die diesbezuiglichen Resultate Spinas wurden in neuerer Zeit von Vieris( ${ }^{4}$ ) vollauf be-

1) 0. Hertwig, Arch. f. mikr. Anat. Bd. 9.

2 , STRICKER, Vorlesungen usw. 1883. S. 351 .

3. Rollet, Bindesubstanzen " in Strickers Handb. d. Gewebelehre. 1868.

4) Vienisa, Experimentelle Untersuchungen über die Regeneration des Sehnengewebes. Virchows Arch. Bd. 125. 1891. 
stätigt, so daß es nicht notwendig erscheint, auf andre Angaben zu rekurrieren.

Gegen die Anwendung der Ergebnisse der Lehre von der Entzündung in der Angelegenheit der Entstehung von Zellen aus der Grundsubstanz können keine Einwendungen gemacht werden. Denn bei der Verwendung eines künstlichen Reizes der entzündlichen Reizung) wird jener Vorgang nur erst auffallend. Es kann doch wohl nicht erwartet werden, daß jemand die Voraussetzung hegt, daß bei der Entzündung Vorgänge von statten gehen, welche sich von analogen physiologischen Vorgängen qualitativ unterscheiden; aus der Grundsubstanz entstehen Zellen, wie an andern Objekten durch direkte Beobachtung bewiesen werden kann, auch im normalen Gewebe, jedoch in einem Grade, welcher der Beobachtung" nur schwer zugänglich gemacht werden kann.

In einer künstlich entzündeten alten Sehne, also einer solchen Sehne, welche wenig Zellen und Ausläufer, dagegen aber viel Grundsubstanz besitzt, beobachtet man also vor allem, daß die vorher flachen Zellen ihr Volumen vergrößern, daß die Ausläufer derselben den Charakter von elastischen Fäden einbiißen und in einer großen Menge auftreten; der Umfang derselben wächst stetig, gleichzeitig vermindert sich die Grundsubstanz, bis schließlich überhaupt fast keine mehr tubrigbleibt. Die Stelle der Fibrillenbündel wird von angeschwollenen Zellausläufern eingenommen, welche später zu den einzelnen Eiterzellen zerfallen.

Die Möglichkeit, daß eine Immigration von Leucocyten vor sich gegangen wäre, wurde durch die Beobachtungen, welche SpINA an Längsschnitten der entzündeten Sehne gemacht hat, ausgeschlossen. An denselben kann nämlich konstatiert werden, daß zur Zeit, in welcher die abgeflachten Sehnenzellen ihren Umfang vergrößern, die Fliigel (primäre Ausläufer) derselben knopfförmige Auschwellungen zeigen. Diese Anschwellungen wachsen und lassen, da sie zu den Ausläufern der zu Kolumnen angereihten Zellen angehören, die gleiche Aneinanderreihung sehen. Nachdem die Schwellung aber einen bestimmten Grad erreicht hat, trennen sich die Knoten von den urspriinglichen Zellen, versehen sich mit Kernen und die Sehne besitzt um eine neue Zellenkolumne mehr. Mehrere nebeneinanderliegende Kolumnen ron dem eben geschilderten Ursprunge entsprechen auf den Querschnitten der Sehne den entzündlichen Zellhaufen.

Aus dem Angeführten geht hervor, daß bei der Sehnenentzuindung viele neue Zellen durch direkte Umwandlung der Grundsub- 
stanz entstehen. Von jedem andern Gewebe der Bindesubstanzengruppe und anch von andern Geweben könnten analoge Beispiele des Schwundes und der Entstehung der Zellen in der Grundsubstanz beigebracht werden.

Doch hat Stricker auch ron dem Corneal-Epithel die Angabe gemacht, von deren Richtigkeit man sich durch längere kontinuierliche Beobachtung leicht zu überzeugen vermag, daß sich die Kittstreifen zwischen den Epithelzellen in Teile der anliegenden Zellen umwandeln und daß neue Kittleisten mitten in den vorhandeneu Zellen entstehen können, so daß die Gestaltung des Epithels, sofern es sich um das Verhältnis zwischen den Zellen und der Grundsubstanz handelt, während des Lebens stetigen Veränderungen unterworfen ist.

\section{Analyse und Feststellung des Problems.}

7. Definition des morphologischen Metabolismus des lebenden Protoplasmas.

Die folgenden Darlegungen sollen sich mit der näheren Analyse der Erkenntnis beschäftigen, welche sich aus der oben angeführten Reihe ron an der lebenden Substanz zu beobachtenden Erscheinungen ergibt. Meiner Ansicht nach stehen nämlich jene Phänomene in einem inneren Zusammenhange, indem sie sich als der morphologisch verschiedentlich verlaufende und ausklingende Ausdruck einer bisher wenn nicht direkt übersehenen, so doch gewiß wenig gewürdigten, Fähigkeit des Protoplasmas darstellen.

Diese Fähigkeit ist nach allem, was mich bis jetzt die Erfahrung gelehrt hat, aller lebenden Substanz, freilich in einem nach Zeit und Ort verschiedenen Grade, gemein.

Die erwähnte Fähigkeit gibt schließlich alle Charaktere eines elementaren Geschehens zu erkennen.

Ich bringe für dieselbe die Bezeichnung: morphologischer Metabolismus des lebenden Protoplasmas in Antrag.

Diese Bezeichnung charakterisiert, wie ich glaube, die Art und Weise des durch die in den Abschnitten 1-6 angefuhrten Tatsachen angedeuteten Geschehens seinem Wesen nach, indem sie zugleich eine bündige Definition desselben involviert.

Mit Bezug auf die Definition des Metabolismus sind weiterhin noch einige Umstände zu erwägen, von welchen ich besonders die nachstehenden erwähne. 
Wie durch die in den Abschnitten 1-6 mitgeteilten Tatsachen gezeigt worden ist, beruht das Wesen des Metabolismus auf der Fähigkeit einzelner morphologischer Bildungen der lebendigen Substanz oder deren Teile, sich in lebende Bildungen von anderm morphologischen Charakter umzuwandeln, welch letztere wiederum imstande sein können andre oder jene ursprünglichen morphologischen Bildungen aus sich herauszubringen. Wiewohl es bei diesen Lmwandlungen offenbar auch zu ehemischen Wandlungen kommt, so kann doch nicht geleugnet werden, daß es sich um Vorgänge handelt, deren Bedeutung vor allem auf morphologischem Gebiete liegt und welche in einigen, besser bekannten Fällen, einen unverkennbar zweckmäßigen Charakter zur Schau tragen. Man kann beobachten, da $\beta$ die lebendige Substanz in bestimmten Fällen Subjekt und Objekt eines Geschehens werden kann, welches mit dem Schwunde vorhandener morphologischer Gebilde - was ich kurz als Morpholyse bezeichnen würde - oder umgekehrt mit deren Ausbildung Morphogenese - verbunden ist, oder selbe zur Folge hat.

Die Strukturelemente des Cytoplasmas oder des Kerns; das Cytoplasma, der Kern, als Ganzes genommen; das Centrosoma; die Grundsubstanz der Gewebe und die Zellen derselben erscheinen somit in bestimmten Fällen als eventuell ${ }^{1}$ ) chemisch abweichend charakterisierte morphologische Formen des ursprünglich »undifferenzierten* Protoplasmas, sie erscheinen nicht als stabile, sondern als dem morphologischen Metabolismus unterworfene Gebilde.

Daher erscheint der morphologische Metabolismus des Protoplasmas durch die Fähigkeit des Protoplasmas zu autonomen, zweckmäßigen morphologischen Umwandlungen definiert.

Aus dem Angefuhrten geht hervor, dab ich unter Protoplasma in Übereinstimmung mit WaLDEYER überhaupt jene Substanz verstehe, welche das Objekt und Subjekt jedes beliebigen vitalen Geschehens bildet und zwar entsprießt diese meine biologische Auffassung des Protoplasmas eben der Riucksicht, welche ich auf die Erscheinungen des morphologischen Metabolismus nehme.

\section{Der morphologische Metabolismus als elementares Geschehen.}

In dem vorhergehenden Abschnitte habe ich mich dahin ausgesprochen, daß der morphologische Metabolismus alle Charaktere eines

1) Darauf koume ich noch in Auschnitt $11 \mathrm{zn}$ sprechen. 
elementaren Geschehens an sich trägt. Ich schreite nunmehr zur Mitteilung der Grinde, auf welche gestützt ich jene Aussage getan habe.

Diesbezüglich ist vor allem zu erwägen, daß sich ein Teil der in den Abschnitten 1-6 durch typische Fälle angedeuteten Vorgänge, nämlich die Morpholyse, an den elementaren Strukturteilen des Cytound Karyoplasmas abspielt, wie aus den Angaben der Abschnitte 1 und 2 hervorgeht. Die betreffenden Teile sind in der Tat Elementarteile, da sie-mit Hinblick auf den Mutterboden, aus welchem ihre morphogenetische Differenzierung vor sich geht, sich entschieden als etwas Neues erweisen, so daß man sie im Sinne der Ausführungen von DrIESCH ${ }^{1}$ ) uber positiv bestimmte Elementarorgane unter einfacher Worteinwechslung als positiv bestimmte Elementarteile bezeichnen kann, welche, wie eben ihr Metabolismus lehrt, im allgemeinen Sinne blok durch ihren Anfang und ihr Ende gekennzeichnet sind, obne andre Differenzen aufzuweisen.

Mit Hinblick auf diese Elementarteile erseheint also der Metabolismus deutlich als ein Vorgang von elementarem Charakter.

Bei einer oberflächlichen Anschauung könnten jedoch bezüglich des Kerns in bezug auf den Zellleib und bezüglich der Zelle mit Bezug auf die Grundsubstanz in unter die Kategorie der in den Abschnitten 5 und 6 hervorgehobenen Erscheinungen fallenden Fällen Zweifel auftauchen, ob diese Gebilde dem Begriffe von positiv bestimmten Elementarteilen entsprechen.

Würde man bei jener Auffassung der Beziehungen des Kerns zum Cytoplasma und der Zelle zur Intercellularsubstanz, wie sie jetzt fast allgemein anerkannt wird, verharren, so könnte der Gedanke, $d a B$ es sich um Elementarteile handelt, freilich überhaupt nicht aufkommen. Meine Auffassung dieser Beziehungen wird jedoch durch die Rticksicht auf die Erscheinungen des morphologischen Metabolismus bestimmt, welche man bisher übergangen hat.

Nimmt man nun von diesem Standpunkte aus die Beurteilung des Verhältnisses des Kerns zum Cytoplasma in Angriff, so müssen vor allem die Ergebnisse meiner Beobachtungen an den Leucocyten beribksichtigt werden, welchen der Wert von Versuchen gebuhrt und durch welche der Beweis geliefert wurde, daß - wenn es nach der Morpholyse zu einer neuerlichen Morphogenese des Kerns kommt,

1) Drresch, Die Lokalisation morphogenetischer Vorgänge. Arch. f. Entw.Mech. 1899. 
der neuaufgetauchte Kern in Gestalt, Zahl und Lagerung von dem untergegangenen völlig differieren kann, so daß er dem Mutterboden gegentiber, aus welchem die morphogenetische Differenzierung desselben geschah, tatsächlich etwas Nenes vorstellt. Noch klarer tritt dies in dem von mir beobachteten und oben mitgeteilten Falle hervor, in welchem der in Teilung begriffene Kern einer Amöbe morpholytisch unterging, der neu entstandene jedoch alle Charaktere eines völlig ruhenden Kerns zur Schau trug. Bei ähnlichen Vergleichen ist es freilich notwendig, von allen Charakteren, welche sonst den Kern auszeichnen, zu abstrahieren, mit Ausnahme der morphologischen Charaktere, da es sich ja um die Charakterisierung von vorwiegend morphologischen Vorgängen handelt. Wenn dabei für jemanden der Umstand, daß der Kern kein morphologisch elementares Gebilde, sondern im Gegenteil ein komplexes ist, ein Hindernis zu bilden scheint, so möge er sich in Erinnerung bringen, daß sich der Begriff' der elementaren Morphogenése, dem wir uns in den vorliegenden Darlegungen zu nähern suchen, selbstverständlich nicht auf das fertige Gebilde (den Effekt), sondern nur auf dessen Entstehung beziehen kann.

Mit Ritcksicht auf diesen Umstand gestatte ich mir neuerlich auf die Resultate der Forschungen Tellyessiczkys, die im Abschnitt 2 zitiert worden sind, hinzuweisen. Aus denselben geht nämlich hervor, daß sich der Kern in einem bestimmten Augenblicke seines Daseins (im Ruhestadium) von dem Cytoplasma morphologisch nur dadurch unterscheidet, daß er von dem letzteren durch eine differente Membran getrennt ist. Es sind dies die Kerne, welche Korschlit als *leer* und mit den Kernfarbstoffen unfärbbar bezeichnet hat. Daß aber die Membran, welche derartige homogene, chromatinfreie Kerne begrenzt, kein stabiles Gebilde zu sein braucht, ist bereits von STricker und Korschelt erwiesen worden.

Die Resultate der Merotomie einzelliger Organismen, welche der Lehre vom morphologischen Metabolismus des lebenden Protoplasmas scheinbar widerspricht, bilden - wie ich sofort zeigen werde eine weitere Stutze meiner These, daß der Kern als positiv bestimmter Elementarteil aufgefaßt werden kann.

Die Morphogenese des Kerns hat man sich gewiß als die Ausscheidung gewisser Protoplasmateilchen und Verbindung derselben zu. einem in bestimmter Weise definierten morphologisehen Gebilde vorzustellen. Nachdem VERWorx u. a. nachgewiesen haben, daB der einer Zelle entnommene Kern einer selbständigen Existenz ebenso 
unfähig ist wie das zuriuckgebliebene Cytoplasma, so muß anerkannt werden, daß bei der Morphogenese des Kerns ans dem Protoplasma gewisse, im Laufe der Morphogenese spezifischen Charakter ${ }^{1 ;}$ annehmende, Teilchen ausgeschieden werden, nach deren Entnahme aus der Zelle der Rest nicht mehr fähig erscheint, sich auf Grund regulatorischer Vorgänge wieder zum Ganzen (der Zelle! zu vervollständigen. In einem solchen Falle treten also Cytoplasma und Kern einander als Objekte von ungleichem Charakter gegenüber.

Wenn es also nach der Morpholyse des Kerns zu dessen Morphogenese kommt, so muß man notwendigerweise roraussetzen, da $\beta$ das undifferenzierte Protoplasma regulatorisch etwas sich Ungleiches herauszubilden vermag, mit andern Worten, daß der fertige, solchermaken entstandene Kern dem Mutterboden gegenuber etwas Nenes vorstellt, d. h. eben ein positiv bestinmter Elementarteil ist.

Die gleichen Griinde, welche ich für die Beziehungen des Kerns zur Zelle angeführt hahe, gelten auch fur die Beziehungen der Zellen zur Intercellularsubstanz, trotzdem der elementare Charakter dieser beiden im Sinne der Morpholyse hauptsächlich nur in der Ontogenese, im Sinne der Morphogenese vorwiegend in pathologischen Fällen, besonders bei der Entzündung, zutage tritt. In diesem letzterwähnten Falle kann sicherlich nicht geleugnet werden, daß wenigstens ein Teil der in der Grundsubstanz anftauchenden neuen Zellen der Grundsubstanz gegenüber als etwas Neues hingestellt werden muß.

Weitere Gründe für die Annahme des elementaren Charakters des morphologisehen Metabolismus des lebenden Protoplasmas werden noch in den Darlegungen der folgenden Abschnitte 9 und. 11 beigebracht werden.

\section{Die Voraussetzungen des Metabolismus.}

Folgt man aufmerksam dem iber die in den Abschnitten 1-6 mitgeteilten Tatsachen Angeführten, so findet man, daß das durch dieselben zum Ausdrucke gelangende Geschehen einen besonderen Charakter an den Tas legt, dessen Wesen sich in den nachfolgenden Umständen spiegelt.

Unter bestimmten Bedingungen tragen die Zellen die Fähigkeit zur Umwandlung in die Grundsubstanz zur Schau; der Zellkern ist imstande sich unter gewissen Umständen zum Cytoplasma umznwan-

1 In morphologischem und chemischem Sinne. 
deln; der Nucleolus ist einer Verwandlung in das Cytoplasma: den Kern, in die Chromosomen, den strukturlosen Kernsaft oder auch in die Spindel fähig; das Centrosoma wandelt sich in undifferenziertes Plasma um; die Spindel transformiert sich zu dem sog. Nebenkern oder zum Centroplasma; die Mitomfäden werden zu Mikrosomen. Bei der Morpholyse der im Cyto- und Caryoplasma differenzierten Mikrosomen und Fäden und überhaupt bei der Morpholyse der Protoplasmastrukturen rersehwindet die geformte Phase des Protoplasmas, das letztere wird homogen, einphasig.

Bei der Morphogenese der Protoplasmadifferenzierungen beobachtet man umgekehrt, daß aus dem undifferenzierten Plasma Mikrosomen, Fäden und kombinierte Protoplasmastrukturen entstehen können; aus der Grundsubstanz Cytoplasma, Zellen, Kerne; aus dem Cytoplasma können hervorgehen das Centrosoma, die Spindel, der Kern, die Grundsubstanz; ans dem Kerne das Centrosoma (sowohl aus dem Chromatin - wie aus dem Achromatinanteil), die Spindel, der Nucleolus, das Cytoplasma; aus dem Centrosoma (Centroplasma) wiederum die Spindel usw.

Des weiteren kann man beobachten, daß einzelne Differenzierungen selbst durch Zusammenwirkung mehrerer andrer Protoplasmaformen zur Entstehung gebracht werden können, z. B. aus dem Körper und dem Kern können das Centrosoma oder die Spindel oder die Grundsubstanz hervorgehen.

Ob die Morpholyse oder die Entstehung der angeführten Differenzierungen etwa in einer bestimmten Reihenfolge geschieht, z. B. nach der Kompliziertheit derselben, ist vorläufig ganz irrelevant und vermag nichts an der Tatsache zu ändern, welche sich aus dem Angeführten ergibt, daß nämlich jedwede Protoplasmad if ferenzierung der Umwandlung in das undifenzierte Protoplasma und dieses letztere selbst wieder einer Umwandlung in jedwede andre Protoplasmadifferenzierung fähig ist, freilich unter der Voraussetzung einer »zweckmäßigen * Beschränkung.

Dies ist gewiß die ganz zweifellose Schlußfolgerung aus der Reihe der oben angeführten Tatsachen.

Der morphologische Metabolismus ist also durch eine besondere Entstehungsweise charakterisiert.

Zur näheren Charakteristik derselben halte ich es für zweckmäßig, auf den von Driesch, freilich zu andern Zwecken geschaffenen Begriff der prospectiven Potenz zurlickzugehen. Driesch 
bezeichnete nämlich als prospective Potenz das, was aus einem bestimmten Teile des embryonalen Körpers im Laufe der Ontogenese werden kann.

Zwar hatte auch Driesch bereits an die Möglichkeit einer ausgedehnteren Benutzung dieses Begriffes gedacht ${ }^{1}$ ), als er davon sprach, daß derselbe durch Anwendung an die Elemente des gegebenen embryonalen Teils an Bedeutung gewinnt und als er anführte, daß der Begriff des Elementes viel weiter ist, als der Begriff der Zelle, und daß dort, wo es sich um ein intracelluläres Geschehen oder um ein Geschehen bei Protisten handelt, die Begriffe der Zelle und des Elementes nicht zusammenfallen.

Doch waren diese Ausfuhrungen Drieschs im ganz allgemeinen Sinne gehalten, denn die Tatsachen, von welchen er ausgegangen ist, boten ihm kein Material zur sachlichen Begründung einer ausgedehnteren Benutzung des Begriffes der prospectiven Potenz.

Um so eher gestatten dies aber die Tatsachen des morphologischen Metabolismus.

Ich nehme an, daß man mir beipflichten wird, wenn jch mir auf Grund der in den Abschnitten 1-6 angeführten Tatsachen das Recht herausnehme, die Vorgänge, um welche es sich handelt, der von DriEsch studierten ontogenetischen Morphogenese gegenüber, als die im eigentlichsten Sinne elementare Morpholyse und Morphogenese zu bezeichnen. Die ontogenetische Morphogenese erscheint mir mit Hinblick auf den morphologischen Metabolismus, als ein allem Protoplasma gemeinsames Geschehen, nur als ein bestimmt charakterisierter Spezialfall der Morphogenese überhaupt, also nur als ein Teil der unter der Bezeichnung: morphologischen Metabolismus zusammengefaßten Phänomene.

Sobald mir der Zusammenhang der im Abschnitte 1-6 zitierten Erscheinungen im Sinne des morphologischen Metabolismus klar geworden ist, wurde mir gleichzeitig auch klar, daß es notwendig ist, zu bestimmen, welche die prospective Potenz der in Frage stehenden Gebilde sei und welche Eigenschaften sie zu erkennen gebe, um zur Formulierung der fundamentalen Voraussetzung jenes Geschehens im Protoplasma zu gelangen.

Der Schluß, zu welchem ich oben gelangt bin, daß nämlich jedwede Protoplasmadifferenzierung der Umwandlung in das undifferenzierte Protoplasma und dieses letztere selbst wieder einer Um-

1. Driesch, Lokalisation usw. S. 69/70. 
wandlung in jedwede andre Protoplasmadifferenzierung fähig ist ist nur eine Analogie des Ausspruches von Driesch, daß sjedes alles kann *. Aus den Tatsachen des morphologischen Metabolismus geht also hervor, daß die morpholytische und morphogene prospective Potenz aller Teile des lebenden Protoplasmas gleich sein muB.

Die prospective Bedeutung derselben hängt offenbar nur von ihrer relativen Lage im ganzen ab (siehe auch Abschnitt 10).

Die eben konstatierte gleichmäßige Verteilung der prospectiven Potenz auf alle Teile des lebenden Protoplasmas bildet die fundamentale Voraussetzung des morphologischen Metabolismus.

Ich verfolge weiterhin nur den Gang von Drieschs Argumentation, wenn ich die Unstände anfuhre, welche diese Äruipotentialität des Protoplasmas ins nähere Licht zu setzen befühigt sind.

So ist es ohne weiteres klar, daß jeder Effekt des morphologischen Metabolismus in einer bestimmten Anzahl und in einer festen Beziehung zu allen uibrigen Effekten zustande kommt, d. i. daß die Differenzierung mit Rücksicht auf die übrigen Differenzierungen geschieht. Mit andern Worten: das Proportionale und Typische des Ganzen wird gewahrt. Das bedeutet, daß das System, in welchem der morphologische Metabolismus zur Geltung kommt, selbst die Art und die Größe der Vorgänge bestimmt, durch welche sich der Metabolismus verwirklicht. Auf diese Weise kommt also auch der primär regulatorische Charakter des morphologischen Metabolismus zum Ausdruck.

Solchermaßen wird es offenbar, daß der morphologische Metabolismus nur auf der Basis möglich wird, daß sich das Protoplasma als ein harmonisch-äquipotentielles system erweist.

Driescr hat den Beweis geliefert, daß die ontogenetische Differenzierung ein harmonisch-äquipotentielles System zur Voraussetzung hat. Er hat diese Schlußfolgerung aus dem Verhalten von Eiern und einzelnen niedrig organisierten Organismen gegenuber von kunstlich gesetzten Störungen abzuleiten vermocht.

Bei den Objekten, welche den Ausgangspunkt meiner Deduktionen bilden, ist es freilich nicht immer möglich, an die Lösung der gesetzten Frage mittels Versuchen zu schreiten und zwar wegen des elementaren Charakters der betreffenden Teile der im Abschnitt 8 nachgewiesen worden ist. Selbstverständlich kann aber solchen, in den Abschnitten 1-6 angefubrten Tatsachen, welche nur durch Be- 
obachtung, nicht aber mit Hilfe des Versuches gewonnen worden sind, kein geringerer Wert, keine mindere Überzeugungskraft imputiert werden. In manchen Fällen müssen nämlich jene Beobachtungen Versuchen gleichgestellt werden, besonders dann, wenn der Morphogenese die Morpbolyse vorausging, was mit Hinblick auf den elementaren Charakter der betreffenden Teile bezüglich der effektiven Wirkung einem kiinstlich gesetzten Defekte gleichkommt. Ein Teil jener Tatsachen wurde aber tatsächlich auf dem Wege des Versuchs eruiert (siehe Abschnitt 3).

Nachdem, den Ausführungen von DrIEsch gemäß, die Charakterisierung eines Lebenssystems als harmonisch-äquipotentielles jmplicite die Behauptung einschließt, daß Differenzierungsgeschehen an ihm Antwortgeschehen sei und weil der Begriff des Antwortgeschehens nach den Darlegungen desselben Autors ein elementarer Begriff ist, so geht aus meinen obigen Darstellungen des weiteren hervor, daß der morphologische Metabolismus als Antwortgeschehen zugleich auch elementares Geschehen sei. Die Konstatierung dieses sei eine Vervollständigung der Deduktionen des Abschnittes 8, welche in demselben keinen Platz finden konnte, weil sie erst das Ergebnis der in diesem Abschnitte niedergelegten Erwägungen bildet.

Zum Schlusse sei noch das Nachfolgende hervorgehoben.

Ich habe die harmonische Äquipotentialität der Protoplasmateile als Voraussetzung des morphologischen Metabolismus bezeichnet. Es liegt an der Hand, daß sie dies tatsächlich ist, denn die Ursachen des Metabolismus sind keine äußeren, sondern innere, so daß die oben erwähnte Eigensehaft des Protoplasmas im Sinne der Terminologie von Driesch als Bedingung des Systems bezeichnet werden kann.

10. Worin beruht das Problem des morphologischen Metabolismus?

Auch in diesem Absatze brauche ich mich nur den Deduktionen Drieschs anzuschließen, welcher das analoge ontogenetische Problem derartig vertieft hat, daß es schwierig ist, etwas Neues hinzuzuftigen. Dieser Umstand ermöglicht es mir aber auch, in dem vorliegenden Kapitel mit der größtmöglichen znlässigen Kürze vorzugehen, obschon in demselben das eigentliche Wesen des vorliegenden Problems gekennzeichnet werden soll.

Der morphologische Metabolismus erscheint als ein zur Entstehung von Verschiedenheiten führendes Geschehen, welche sich 
durch ein typisches Verhältnis zum Ganzen offenbaren. Dieses Verbältnis wird durch die Lokalisation der entstandenen Verschiedenheit bestimmt.

Der morphologische Metabolismus ist ein elementares Geschehen. In dem Begriffe eines elementaren morphogenen Vorganges ist jedoch nach DRIEscrr nicht der Begriff der Form, sondern nur der Begrift des Bestehens einbegriffen. Der Begriff der Form taucht erst dañn anf; wenn sich die Resultate mehrerer elementarer morphogener Vorgänge vereinigen. Die Spezifität der Form (Differenzierung) ist nach Dricsch eine Folge der Lokalisation ihrer Entstehung.

Die Lokalisation des morphogenetischen Geschehens ist es, wenn man die zitierten Lmstände erwägt und auf die in der vorliegenden Abhandlung diskutierten Vorgänge appliziert, also a uch bei dem morphologischen Metabolismus, was eine Lösung herausfordert.

Nach Driesch erscheint aber die Lokalisation ontogenetischer Vorgänge in dem Falle als ein besonderes Problem, wenn sich ein harmonisch-äquipotentielles System mit primärer Richtungsorganisation unter Ausschließung äußerer Ursachen differenziert. Lin angemessenes energetisches Medium, welches die Erhaltung des Lebens ermöglicht, wird freilich voransgesetzt.

Versuchen wir das eben Ausgesagte auf den morphologischen Metabolismus anzuwenden, so nehmen wir wahr,

1) daß das Substrat, in welchem er geschieht, sich als ein harmonisch-äquipotentielles System darstellt;

2) daß mit Hinblick auf die Äquipotentialitit der Teile dieses Systems natïlicherweise ein primäres Gerichtetsein derselben angenommen werden muk, welches durch eine aubere Ungleichheit gekennzeichnet ist, da ja sonst jedes Differenzieren unmöglich wiire;

3. daß - sofern es sich um die Ursachen der Differenzierung handelt - bereits der erste Blick offenbart, daß von einer morphogenetischen Wirkung der bekannten formativen Reize selbstverständlich keine Rede sein kanu.

Daraus ergibt sich, daß das Problem des morphologischen $\mathrm{Me}$ tabolismus mit dem Probleme der Lokalisation ontogenetischer Vorgänge, welches Driesch zum Gegenstande seines Studiums gemacht hat, analog ist.

Weil die Differenzierung in einem harmonisch-äquipotentiellen 
Systeme unter Ausschluß der Wirksamkeit äußerer Reize geschieht, erscheint DRIEsch die Lokalisation des morphogenetischen Geschehens mit Hinblick auf den Umstand, daß sie anf die Ausbildung eines typischen Ganzen gerichtet ist, auf Grund der aus den anorganischen Disziplinen bekannten Gesetzmäßigkeiten unerklärbar. Er hält sie für eine neue Art elementarer Gesetzmäßigkeit, für den Ausfluß einer besonderen vitalistischen Causalität, welche nur der lebenden Sabstanz eigen sein soll, weil "sich prinzipiell keine mit chemischphysikalischen Mitteln gefertigte maschinelle Einrichtung ersinnen läßt, anf der als Basis sich das vorliegende Geschehen abspielen könnte «.

Es ist kein maschinelles Geschehen, weil bei einer Maschine die Regulation durch Ersatz, nicht aber durch eine Modifikation des Geschehens erreicht wird, wie in dem vorliegenden Falle.

$\mathrm{Zu}$ diesen Schlußfolgerungen Drieschs werde ich im Abschnitte 12 Stellung nehmen.

\section{Versuch einer Lösung.}

11. Die Ursachen des morphologischen Metabolismus.

Auf Grund des in den friiheren Abschnitten Dargelegten kann kein Zweifel mehr darïber bestehen, daß der morphologische Metabolismus gegenuber der ontogenetischen Morphogenese ein durchaus elementares Geschehen darstellt, denn er tritt nicht bloB bei Vorgängen auf, welche mit der Entwicklung des Individuums zusammenhängen, sondern auch bei solchen, die im Substrate der Entwicklung selbst oder aber in einem Protoplasma, dessen Entwicklung schon seit langem vollendet ist, vor sich gehen; außerdem handelt es sich sehr oft um intracelluläres Geschehen (siehe auch Abschnitt 8).

Der elementare Charakter des morphologischen Metabolismus geht jedoch auch noch aus dem Nachfolgenden hervor.

Bei dem Studium der in den Abschnitten 1-6 angedeuteten Erscheinungen zeigt sich nämlich, $d a B$ eine jede der angeführten Metamorphosen des Protoplasmas gleichzeitig mit einer chemischen Umwandlung desselben einhergeht.

Bei der Umwandlung ganzer Zellen in die Grundsubstanz (z. B. bei der Entwicklung des Knorpels) und umgekehrt, ist dies obne weiteres klar. Dasselbe gilt auch ron der Lmwandlung des Kerns in das Cytoplasma oder von dem umgekehrten ProzeB.

Weniger auffallend sind diese Wandlungen sofern es sich um 
Metamorphosen von Kern- und Cytoplasmastrukturen handelt. Dies hat seinen Grund in dem Umstande, daß unsre Kenntuis der chemischen Zusammensetzung dieser Teile sehr liickenhaft ist. Trotzdem besitzen wir gewisse Hinweise, die - wenn sie auch kein Bild der chemischen Umlagerungen, welche hier von'statten gehen, geben - doch bezeugen, daß es sich um solche handelt. Die erwähnten Hinweise ergeben sich aus der Benutzung der vitalen Neutralrotfärbung, von weleher ich bewiesen habe, daB sie chemischer Natur ist ${ }^{1}$ ). Wenn bei der Morpholyse der Granula und Cytoplasmafäden auch die vitale Färbung derselben schwindet, wenn die letztere mit der Morphogenese derselben wieder auftritt, so ergibt sich daraus mit Klarheit, daß diese Vorgänge mit chemischen Veränderungen des Protoplasmas verbunden sind, welche zum Schwunde oder zur Aktivierung der Färbung führen. Der Schluß, daß die Cytoplasmastrukturen von dem einphasigen Protoplasma chemisch verschieden sind, erscheint daher vollkommen berechtigt.

Die gleiche Schlußfolgerung gilt, wie leicht nachgewiesen werden kann, auch für die Differenzierungen des Karyoplasmas.

Es wird z. B. anerkannt, daß in gewissen Lebensabschnitten des Kerns das Chromatin der Kernschleifen schwindet und nach demselben nur das Linin, die Grundsubstanz des Chromatins, zurìckbleibt, welche außer der Unfärbbarkeit mit basischen Farbstoffen auch durch Unterschiede von ganz zweifellos chemischer Natur charakterisiert ist. Bereits HäckER hat die Vermutung ausgesprochen, daB die Bedeutung der persistierenden Chromosomen nicht im Chromatin, sondern im Gegenteil im achromatischen Substrate derselben beruht. Es ist aber freilich zweifelhaft, ob man die chromatinfreien Gebilde noch als Chromosomen bezeichnen darf, da das Chromatin, wie Fraxk Schwarz gezeigt hat, kein rein morphologiseher Begriff ist, sondern auch chemischen Sinn besitzt, und zwar einen solchen, der von dem des Linins vielfach abweicht. Im tibrigen schwinden ja, wie aus den Beobachtungen Tellyesniczkrs hervorgeht, die Chromosomen nicht bloßs so, daß sie sich nur ihres Chromatins entblößen würden, sondern es wandelt sich auch das nach ihneu zurückgebliebene Liningerïst schließlich in den $»$ Kernsaft $«$ um, der ja nach den Ausführungen des letztgenannten Autors der wichtigste und allein koustante Bestandteil des Kerns ist.

1. Rủžčks, Zur Theorie der vitalen Färbung. Zeitschr. f. wiss. Mikrosk. XXII. 1900̃. 
Vom Kernsafte - Paralinin - ist aber zu dem Plastin des Zelleibes nur ein kleiner chemischer Schritt, wie neben andern auch aus dem Limstande $\mathrm{zu}$ ersehen ist, daß diese beiden Substanzen der kïnstlichen Magensaftverdaunng unterliegen.

Aus dem Angefuihrten ist zu entnehmen, daß Frank Schwarz' morphologisch-chemische Unterscheidung der Zellbestandteile eine sachliche Basis besitzt. Daß durch dieselbe keine chemischen Einheiten, ja selbst keine chemischen Verbindungen unterschieden werden und auch nicht unterschieden werden können, ist begreiflich, weil es sich um stetig ineinander übergehende Gebilde handelt. Charakteristisch ist jedoch, daß bei dieser Lnterscheidung der morphologische Begriff mit gewissen chemischen Charakteren untrennbar verbunden erscheint und daB diese Untrennbarkeit auch in den Fällen des morphologischen Metabolismus typisch gewahrt bleibt, wodurch eben die Frank Schwarzsche Unterscheidung für die Erforschung dieses Geschehens eine besondere Bedeutung erhält.

Wie weit jene Unzertrennlichkeit der chemischen und morphologischen Charaktere einer Differenzierung reicht, - ich möchte derselben durch Bezeichnung dieser Vorgänge als morphochemische 1) kurzen Ausdruck verleihen - zeigen meine nachstehend mitgeteilten Beobachtungen, deren Resultate auf die Geltung eines allgemeinen Gesetzes hinzuweisen scheinen.

Die nachfolgenden Resultate ergaben sich mir bei dem Studium der Sporenbildung des Milzbrandbacteriums.

Von diesem Bacterium babe ich, wie bekannt, den Beweis geliefert, daß es durchgehends ans Nuclein zusammengesetzt ist. Die Frage lag nahe, wie sich zu dieser Tatsache die Produkte der vegetativen bakteriellen Individuen: die Sporen verhalten.

Bevor ich jedoch an die Beantwortung dieser Frage schreite, erlaube ich mir auf zwei neuere Arbeiten hinzuweisen, deren Resultate ich als Bestätigung meiner Behauptung von der Nucleinnatur der Substanz, aus welcher die Bakterien bestehen, anzusehen, wohl das Recht habe.

Dietrich und Liebermeister ${ }^{2}$ ) hahen in den Milzbrandbakterien Körnchen beobachtet, welche sich mit Indofenolblau färbten und welche ihrer Vermutung nach den molekulären Sanerstoff der Luft

1) Vermutlich wird es mir möglich sein. über dieselben neue Beobachtungen in Bälde zu publizieren.

2) Dietrich und Liebermeister. Sauerstoff übertragende Körnchen in Milzbrandbacillen. Centralbl. f. Bacter. 32. I. Orig. 1902. 
za aktivieren haben, was sie aus der eben erwähnten Tinktion abgeleitet haben. Doch stellte sich die Färbung jener Körnchen auch dann ein, wenn dieselben durch Hitze abgetötet worden sind. Sowohl aus der Beschreibung der Autoren, wie auf Grund einer Wiederholung ihrer Versuche meinerseits glaube ich schließen zu dürfen, daß die von ihnen beobachteten Körnchen mit denjenigen, welche ich als Bestandteil der verschiedensten Protoplasmastrukturen der Bakterien beschrieben habe, identisch sind, freilich -- wie ich unten zeigen werde - unter bestimmten Bedingungen. Mit bezug auf diese Identifizierung wird es sicherlich von Interesse sein, die Resultate der mikrochemischen Untersuchung der Körnchen, welche die erwähnten Forscher unternommen haben, kennen zu lernen. Da uberraseht mich vor allem der Kommentar, mit welehem DreTrich und LIEBERMEISTER die Tatsache, daß die erwähnten Körnchen durch Einwirkung des Magensaftes nicht verändert werden, begleiten, indem sie den Zusatz machen * selbst wenn der ganze Bacillenleib aufgelöst ist, höchstens vielleicht noch die etwas widerstandsfähigere Membran übrig geblieben ist . Es ist mir nämlich durchaus nicht klar, woraus die vollständige Auflösung des Bakterienkörpers erschlossen worden ist, wenn - wie ans dem Zitate zu ersehen - die angeführten Körnchen und die Membran zugegen waren. Da ich die Netzstrukturen, deren Bestandteil die besprochenen Körnchen bilden, in den der Magensaftverdauung unterworfenen Bakterien noch am 8. Tage, die übrigen Bestandteile der Bakterien aber selbst noch am 50. Tage der Safteinwirkung vorzufinden vermochte, so muß ich den Schluß ziehen, daß der oben zitierte Ausspruch Dietrichs und LiEBeRmeisters nur einer vorgefaßten Meinung tuber die celluläre Natur der Bakterien seine Entstehung verdankt. Dies mein Urteil steht auch mit den Ergebnissen jener Autoren iber die Chemie der von ihnen beobachteten Körnchen in vollkommenstem Einklange.

Sie widersprechen vor allem der Behauptung Grimres, nach welcher die Körnchen Fettröpfchen sein sollten, obwohl sie mit Sudan III eine Färbung derselben erzielt haben, indem sie anfuhren, daß die letztere auch dann zustande kommt, wenn man die Körnchen mit Äther und Alkohol oder mit Chloroform extrahiert.

Trotzdem halten sie dieselben für Reservestoffe »wenn anch nicht von der gewöhnlichen Art«. DaB es Nucleine wären, meinen sie nicht, indem sie auf die Unveränderlichkeit der Körner in verdünnten Alkalien, Essigsäure und Mononatriumphosphat hinweisen. Doch geben sie zu, daß die Substanz jener Körnchen den Nucleinen nahe steht. 
Der Ansicht, daß die in den Bakterien enthaltenen Körner Reservestoffe sind, begegnen wir auch in den Ausführungen von Meyer ${ }^{1}$ ). Demselben zufolge ist es möglich, daß das Volutin (so benennt er die Substanz jener Körner, welche er außer bei den Bakterien auch bei einer Reihe niederer Pflanzen konstatieren konnte) zu den Eiweißstoffen gehört und eine bedeutende Quantität von Nucleinsäureverbindungen enthält. Trotzdem erklärt er die Volutinkörner für Reservestoffe, indem er zugunsten dieser Ansicht anführt, daß sie sich in keimenden Bakterien gewöhnlich zugleich mit typischen Reservestoffen (Glykogen, Fett) vorfinden, daß ihre Zahl vor der Sporenbildung am größten ist und $\mathrm{da} B$ sie bei derselben gerade so wie Fett und Glykogen verbraucht werden.

Von den Ausführungen Meyers erscheint mir die von ihm festgestellte Tatsache, daß die besprochenen Körner eine reichliche Menge Nuclein enthalten, von viel größerem Werte zu sein, als seine Deutung derselben als Reservestoffe. Denn jene Tatsache stimmt völlig damit überein, was ich über die chemische Zusammensetzung der morphologischen Komponenten der Bakterien in Erfahrung gebracht habe, während für die Deutung der Körner als Reservestoffe MEyen nur Analogien beizubringen in der Lage war, welche freilich mit einem Beweise nicht identifiziert werden können.

Überhaupt scheint mir einer ähnlichen Behauptung vor allem die für das Nuclein charakteristische relative Stabilität des Molekiils im Wege zu stehen. Bekanntlich erleidet das Nuclein der Zellen von einer protrahierten Hungerung ausgesetzten Tieren keine Verminderung.

Was weiterhin die Gegenwart der Nucleinkörner in keimenden Stäbchen, ihre Anhäufung zur Zeit der Sporulation und ihren Verbrauch bei der Ausbildung der Spore anlangt, so können diese Tatsachen im Einklang mit meinen Angaben von den chemischen Verhältnissen der Bakterien erklärt werden, ohne daß man die erwähnten Körner für Reservestoffe zu halten gezwungen wäre. Aus dem nachstehend Mitgeteilten wird sich im Gegenteil ergeben, daß dieselben tatsächliche Differenzierungen der lebenden Substanz sind.

Auf Grumd des Angeführten kann also als sichergestellt gelten, daß sämtliche Komponenten des Milzbrandbacteriums Nucleinsubstanzen entsprechen.

1. Meyer. Orientierende Versuche über Verbreitung, Morphologie u. Chemie des Volutins. Bot. Ztg. 62. 1904. 
Es wird also die Frage nicht als überflüssig erscheinen, ob dieselben eine Differenzierung im Sinve der Ausführungen von Frank ScHWarz zu erkennen geben. Die Entscheidung dieser Frage ist, wie ich bereits erwähnt habe, für den morphologischen Metabolismus und zwar mit Hinsicht auf die an anderu Objekten konstatierten Umwandlungen des Chromatins, von Bedeutung.

Zum Zwecke meiner Untersuchungen habe ich auf mit einigen Tropfen Bouillon benetzte Agarplatten Milzbrandbakterien ausgesät, um die Sporulation zu beschleunigen. Von dem auf diesen Platten aufgewachsenen Material brachte ich je einige Platinösen in Uhrgläser, welche mit den notwendigen Reagentien gefüllt waren. Nach Ablauf von verschiedenen Intervallen wurden sodann aus dem Inhalte der Uhrgläser mikroskopisehe Präparate gewöbnlicb in der Weise angefertigt, daß ich das Tröpfchen am Objektglase lufttrocken werden ließ, mit der Fixierungsflüssigkeit - als welche ich ein Gemisch von gleichen Teilen konzentrierter wässriger Sublimatlösung und $1 \%$ iger Chromsäure benutzte - übergo ; darauf wurde mit Wasser abgespult, um die herauskristallisierten Reste der zu den Reaktionen gebrauchten Salze zn entfernen und das Präparat auf 17 bis 24 Stunden in die obgenannte Fixierungsflüssigkeit übertragen. Nach Beendigung der Fixation wurde mit Wasser abgespült und zu einem Teil mit $1 \%$ iger wässriger Fuchsinlösung, zum andern mit $1 \%$ iger wässriger Chinablaulösung gefärbt; in einzelnen Fällen kam die Heidenhaissche Hämatoxylinlacktinktion in Verwendung.

Ich habe besonders auf die Wirkung der folgenden Reagentien mein Augenmerk gerichtet: $20 \%$ iges Kochsalz, konzentrierte Lösungen von Magnesiumsulfat, Ferrocyankalium, Kupfersulfat, $5{ }_{10}^{0}$ ige Lösung von salpetersaurem Natron, $1 \%$ ige und $5 \%$ ige Monokaliumphosphatlösung.

Meine Versuche ergaben das folgende Resultat. Ich fuihre nicht alle Versuche an, sondern nur Beispiele derselben, da die Ergebnisse iubereinstimmen.

20\% ige NaCl-Lösung (Dauer der Einwirkung 31/2 Stunde bei einer Temperatur von etwa $18^{\circ} \mathrm{C}$. . Die Stäbchen erscheinen vielfach geschwollen, sind in der Regel sehr schlecht färbbar. Die Sporen und andre kleinere Körner treten als glänzende, ungefürbte Körper scharf hervor.

(Dauer der Einwirkung 5 Tage im Thermostat bei $37^{\circ} \mathrm{C}$. .). Das Präparat enthält sehr wenige ganze Stäbchen. Stellenweise liegen 
Haufen ron intensiv mit Fuchsin färbbaren Körnern, oft liegen die Kürnchen so nebeneinander, daß man daraus schließen kann, daß da ein Bacterium bestanden hat, da die Gruppierung der Körnchen die Form und die annähernden Dimensionen eines solchen bewahren. Wo ein Stäbchen besser konserviert blieb, erscheint die in demselben enthaltene gefärbte Substanz unregelmäßig angenagt. Die Sporen ohne sichtbare Veränderung gut sichtbar.

Konzentrierte Lösung ron Magnesiumsulfat (Dauer der Einwirkung $3 \frac{1 / 2}{2}$ Stunden. Die Stäbchen sind geschrumpft, bedeutend enger; obwohl sie sich im großen und ganzen ziemlich intensiv färben, vermag man doch einen Abgang der fürbbaren Substanz zn konstatieren; einzeine Anthraxfäden sind fast ungefärbt. In den ungefärbten Fäden kamn an den Innenkörpern ein enger, verschieden breiter gefärbter Saum beobachtet werden, welche Erscheimung wahrscheinlich mit dem Schwunde der färbbaren Substanz zusammenhängt. Die Sporen sind ungefärbt, glänzend. - (Daver der Einwirkung 5 Tage im Thermostati. Die Stäbchen tragen zum Teil Schrumpfung, zum Teil und dies in der Mehrzahl der Fälle, eine ganz bedeutende Schwellung zur Schau. Die färbbare Substanz erscheint in Form verschieden intensiv gefärbter Fragmente. Es finden sich Stäbchen vor, welche sich nicht mehr ganz färben, sondern eine Verengung der fürbbaren Substanz aufweisen; dieselbe kann auch unebene Ränder zeigen; in andern sieht man entweder solide oder vacuolisierte Schollen der färbbaren Substanz, welche in Größe und Anzahl variieren können. Dabei kann die Färbung der Stäbchen verschieden intensiv sein, so daß man fast normal gefärbten, aber auch nur blaß rosa angehauchten, kaum nnterscheidbaren Schatten begegnen kann, dazwischen aber allen Übergängen. Es kommen auch Stäbchen vor, welche überhaupt keine färbbare Substanz mehr aufweisen. Oft erscheinen sämtliche Individnen mit feinen nicht ganz gleich großen Körnchen erfüllt. Die Innenkörper sowie die Sporen heben sich, so weit vorhanden, klar ab. In Stäbchen, welche die färbbare Substanz fast gänzlich entbehren, erscheinen die letzten Reste derselben in Form von verschieden starken Hüllen der Innenkörper.

Konzentrierte Ferrocyankaliumlösung. (Einwirkungsdauer $3_{1}^{1}, 2$ Stunden). Die Stäbchen sind eher geschwollen und färben 
sich nur sehr blaß. Die färbbare Substanz erscheint in verschiedener Gestalt; die Sporen sind ungefärbt, gut sichtbar.

- (Dauer der Einwirkung 5 Tage im Thermostat). Die Stäbchen sind sehr verengt, färben sich stellenweise schlecht, was besonders an mit Karbolchinablau gefärbten Präparaten deutlich zutage tritt.

Konzentrierte Kupfersulfatlösung (Einwirkungszeit 31/2 Stunden). Die Stäbchen sind geschrumpft; im übrigen gleicht das Bild demjenigen nach der Ferrocyankaliumwirkung. Die färbbare Substanz gibt verschiedene Gestaltung zu erkennen, angefangen von unregelmäßigen Rändern bis zur Füllung der Stäbchen mit groben Schollen.

(Dauer der Einwirkung 5 Tage im Thermostat). Die schlechte Färbbarkeit ist hier allgemein. Die ungefärbten Sporen heben sich scharf $a b$.

$5 \%$ ige Lösung von salpetersaurem Natron (Dauer der Einwirkung $3 \frac{1}{2}$ Stunden!. Die Stäbchen sind zum Teil geschwollen, zum Teil geschrumpft, die färbbare Substanz ist in vielen nicht mehr nachzuweisen, in fast allen erscheint sie sowohl der Menge, als auch der Färbbarkeit nach reduziert zu sein. Auch hier können die letzten Reste derselben als Hüllen der Innenkörper nachgewiesen werden. Die Involutionsformen zeichnen sich insgesamt durch eine intensivere, ja nahezu normale Färbung ans. Die Sporen ungefärbt, gut sichtbar.

- (Einwirkungszeit 5 Tage im Thermostat). Die Stäbchen sind gleichfalls teilweise geschwollen, teilweise geschrumpft. Der Abgang der färbbaren Substanz ist durchgehends bemerkbar. In vielen, der diffusen färbbaren Substanz gänzlich entblöBten, Stäbchen sind sehr feine, rötlich (von Fuchsin?) angehauchte Körnchen enthalten, welche in zahlreichen Individuen zu alveolären oder spiralförmigen Strukturen angeordnet sind. Die ungefärbten Sporen treten deutlich hervor.

1\% ige Monokaliumphosphatlösung (Dauer der Einwirkung $3^{1 / 2}$ Stunden). Die geschrumpften Individuen enthalten die färbbare Substanz in Form von in der Längsachse der Bakterien gelagerten Stäbchen, welche unregelmäßige Ränder anfweisen oder in Gestalt von verschieden großen und unregelmäßig geformten Schollen. Viele Individuen enthalten entweder keine färbbare Substanz oder nur in der allernächsten Umgebung der Innen- 
körper. Die Involutionsformen lassen anch hier eine intensivere; bis fast normale Färbbarkeit erkennen. Die ungefärbten Sporen sind deutlich wahrnehmbar.

(Einwirkungszeit 5 Tage im Thermostat). Die oben angedeuteten Veränderungen sind auch hier in großem Maßstabe zu konstatieren. Die färbbare Substanz gewinnt durch Unregelmäßigmachung der Ränder phantastische Formen, die um so auffallender erscheinen, wenn sie die Hülle der unveränderten Sporen oder Innenkörper bilden. In sporenfreien Fäden ist die Fragmentierung der färbbaren Substanz und zugleich die Verkleinerung der durch diese Fragmentation entstandenen Schollen klar zu konstatieren. In einem fortgeschrittenen Stadium enthalten die Stäbchen bis auf unbedeutende, verschieden große Schollchen, Körnchen und auf die Hüllen der Innenkörper nichts von der färbbaren Substanz mehr.

50 igige Monokaliumphosphatlösung wirkt in analoger Weise, wie die vorhergehende Lösung.

Die geschilderten Versuchsergebnisse gestatten nur eine Interpretation und zwar im Sinne des Schwundes der in den Bakterien enthaltenen färbbaren Substanz. Vergleicht man nun dieses Resultat mit dem über die färbbare Substanz der Kerne ron pflanzlichen und tierischen Zelleu Bekannten, so springt sofort das analoge Verhalten beider in die Augen, so daß man, mit Rücksicht auf die ibrigen Kongruenzen, zu der Schlußfolgerung gedrängt wird, daß sich die farbbare Substanz des Milzbrandbacteriums der Einwirkung der obangefuhrten Agentien gegenüber in derselben Weise wie das Chromatin der Zellkerne verhält. In diesem Sinne ist das Resultat der zitierten Versuche als eine weitere Vervollständigung meines Beweises, daß die das Anthraxbacterium zusammensetzende Substanz, Kernsubstanz ist, anzusehen.

Von Wichtigkeit erscheint jedoch der nachfolgende Umstand. Bereits aus der $31 / 2$ stündigen Einwirkung der obzitierten Chemikalien geht nämlich klar hervor, noch klarer aber ans der 5 tägigen Einwirkung bei erhöhter (Thermostat-) Temperatur, daß dieselben auf die mit wässrigem Fuchsin färbbare Substanz und auf die Sporen in verschiedener Weise einwirkt. Während nämlich jene Substanz schwindet, bleiben die Sporen unverändert.

Dieser Umstand ist aber rom cytologischen Standpunkt aus sehr interessant. 
Denn, während nach den Angaben Frank Schwarz' das Kernchromatin von den obangeführten Agentien anfgelöst wird, bleibt das Linin von denselben unangetastet. Aus dem Umstande, daß die Sporen von ihnen nicht verändert werden, ergäbe sich - da sie auch der Wirkung des künstlichen Magensaftes nicht unterliegen der Schluß, daß die Sporen aus Substanzen bestehen, welche dem Linin entsprechen.

Da die Sporen das Produkt von vegetativen Stäbchen sind, das direkt aus der Substanz der letzteren entsteht, so $\mathrm{muß}$ weiterhin geschlossen werden, daß die Sporen durch eine Umwandlung der Chromatinsubstanz der Bakterien zustande kommen. Denn die sporenfreien vegetativen Stäbchen verlieren, wie meine obangefiuhrten Versuche zeigen, durch die Einwirkung der genannten Reagentien völlig ihre färbbare Substanz, so daß sie wie leer aussehen; somit muß die dem Kernchromatin entsprechende Substanz den Hauptbestandteil derselben ausmachen.

So erscheint das Milzbrandbacterium als ein Objekt, wie man schwerlich ein zweites finden würde, um die vitalen Tmwandlungen des Chromatins daran studieren zu können, die hier in einer, von keinen störenden Einflissen komplizierten Reinheit zutage treten.

In welcher Weise die Sporen durch Umwandlung der chromatischen Substanz der Bakterien entstehen, lehren die nachfolgenden Umstände.

Erinnert man sich der Angaben von Dietrich und Lieberuieis'Ter über die chemische Zusammensetzung der mit Indophenolblau färbbaren Granula, so findet man, daß sie nicht verändert werden durch die folgenden Reagentien: 5\%, ige Kalilauge, 10\%iges Mononatriumphosphat, verđünnte Essigsäure, Eisessig und Magensaft.

Um festzustellen, wie sich diese Körnchen in Präparaten von Milzbrandbakterien verhalten, welche der Einwirkung von chromatinolytischen Stoffen ausgesetzt worden waren, habe ich die Färbung mit Dimethylparaphenylendiamin + $\omega$-Naphthol an Bakterien rorgenommen, welche 5-6 Stunden in 5\% igem salpetersaurem Natron, konzentriertem Kupfer- und Magnesiumsulfat, konzentriertem Ferrocyankalium oder $20 \%$ irem $\mathrm{NaCl}$ verweilt haben, da ich festgestellt habe, daß nach Verlauf dieser Zeit die Chromatinolyse schon stark fortgeschritten ist.

Es zeigte sich, daß die solchermaßen präparierten Bakterien einige Partien enthalten, welche sich mit dem Indophenolblau färben and zwar: kuglige, beliebig gelagerte und in verschiedener Anzahl 
auftretende Körnchen, weiterhin die Innenkörper, die letzteren in der Weise, daß das Ectogranulum meistens schwächer als das intensiv gefärbte Entogranulum gefärbt erschien; schließlich nahm ich auch eine mittelstarke Färbung der Sporen wahr, welche sowohl in Stïbchen eingeschlossene, als auch freie Sporen betraf.

Da eine weitere Verfolgung dieser Verhältnisse ein tieferes Eindringen in die Art der Sporenbildung versprach, als es bis jetzt möglich gewesen, so babe ich Versuche vorgenommen, bei welchen ich die Indophenolblautinktion mit der Fuchsinfärbung zur gleichzeitigen Anwendung gebracht habe.

Dabei ging ich in der Weise vor, daß ich eine $10_{0}$ ige $W$ asserlüsung von Fuchsin in dünner Schicht auf einem Teil des Deckglases eintrocknen ließ, anf einen andern Teil aber je einen Tropfen Dimethylparaphenylendiamin und $a$-Naphthol brachte, die ich unter gleichzeitigem Zusatz eines Kulturteilchens so vermischte, daß der gebildete Tropfen auf die trockene Fuchsinschicht reichte und dieselbe auflöste. Sodann brachte ich das Deckgläschen auf ein ansgehöhltes Objektglas, damit die Luft Zutritt hätte.

Ganz wie ich erwartet habe, stellte sich bei vielen Bakterien eine Doppelfärbung ein. Und zwar färbten sich: die Umrisse des Bacteriums, die Zwischenwände unvollkommen abgetrennter Bakterien, die Netzstrukturen der Bakterienkörper und die Ectogranula in rotem, die Entogranula und große, sporenähnliche Körper, außerdem noch eine Anzahl von Granula in blauem Tone.

Dieses Resultat meiner Benutzung jener zweier Färbungsverfahren gab Hoffnung, daß es auf diesem Wege möglich sein wird, die Wandlungen festzustellen, denen die einzelnen gefärbten Teile im Laufe der Sporenentwicklung unterliegen.

Aus der kongruenten Färbung der Sporen und der nach dem Verfahren von Dietrich und Liebermeister färbbaren Körnchen, die sich aus meinen Versuchen ergeben hat, weiterhin aus dem kongruenten Ausfalle der chemischen Reaktionen bezüglich dieser beiden Gebilde, den ich schon früher erwähnt habe, ging hervor, daß es sich vor allem um die Verfolgung der Schicksale der mit Indophenolblan färbbaren Körner handelt.

Mit Bezug darauf habe ich vor allem zu erinnern, daß sich in keimenden Sporen nach Preisz ${ }^{1}$ ) ein Körnchen befindet, welches die-

1) Preisz, Studien über Morphologie und Biologie der Milzbrandbacillen. Centralbl. f. Bacter. I. 35. 1904. 
ser Autor als den Sporenkern ansieht und das sich mit einer gewissen Menge Plasma von den ubrigen Stäbchen an einem der Pole durch eine Zwischenwand absondert. Dieser Kern schwindet jedoch in der Sporenanlage, wobei sich die letztere in einem bestimmten Zeitpunkte dunkler färbt, als der tibrige Bacillus; darauf erlischt die Tinktionsfähigkeit und die Substanz der Anlage wird stark lichtbrechend und glänzend, zugleich grenzt sie sich immer schärfer ab.

Von den Sporen des Milzbrandbacteriums hat bereits lange ror Preisz Catterina 1) auf Grund einer besonderen Tinktionsmethode die Angabe gemacht, daß sie ein färbbares Körnchen, seiner Deutung nach gleichfalls den Kern, enthalten, das sich hei der Keimung direkt teilt.

Dariber, ob die von Catterina und Preisz beobachteten Körnchen in den Sporen Kerne sind, kann nummehr auf Grund meiner Arbeiten ein definitives Urteil und zwar in negativem Simne abgegeben werden, was aus dem Verhalten der Bakterien und Sporen gegenüber der kiunstlichen Magensaftverdauung hervorgeht.

Diese Erkenntnis macht eine andre Deutung des färbbaren Kornes der Sporen zur Notwendigkeit.

Behalten wir vor allem die nachstehenden Umstände, auf welche bereits Preisz aufmerksam gemacht hat, im Auge.

I. Selbst in den jüngsten Sporenanlagen ist »der Kern« oder wie ich ihn besser bezeichnen will: das Chromatinkorn stets schon enthalten und erreicht daselbst manchmal Dimensionen, welche der *Kern" des Bacteriums nie erreicht. Preisz gibt selbst zu, daß er nicht imstande war, den Übertritt des »Bacteriumkerns ; in die Spore direkt zu beobachten, sondern daß er auf denselben aus der Ähnlichkeit beider und aus dem Umstande schloB, daß Bakterien mit Sporenanlage den $:$ Kern* oft nur in dieser Anlage, nicht außerhalb derselben, besitzen.

II. Zweitens ist darauf zu achten, daß in der Spore späterhin kein »Kern« mehr nachgewiesen werden kann. Preisz glaubt dies in der Weise erklären zu dürfen, daß der »Kern" von dem färbbaren Plasma der Sporenanlage entweder rerdeckt sein kann oder daß es in der Entwicklung des Kerns eine Periode geben kann, in welcher die ỉblichen Färbungsmethoden zur Kenntlichmachung desselben nicht genügen.

1) Catterisa, Ric. sull intima struttura delle spore dei batteri. Atti della Soc. veneto-trent. III. 1898. 
III. Schließlich ist hervorzuheben, daß in der keimenden Spore das in der reifen und freigewordenen Spore abwesende Chromatinkorn wiederum leicht nachgewiesen werden kann.

Es ist klar, daß diese drei Tatsachen ein schweres, wenn nicht überhaupt unlösbares Rätsel bilden, solange man auf dem Boden der cellulären Theorie der Bakterien stehen bleibt. Es ist jedoch interessant, daß Forscher, die außer stande sind sich vorzustellen, daß es ein kernloses Protoplasma geben könnte, bei den Sporen zur Anerkennung einer Ausnahme gezwungen sind. Man kann sich nämlich der Tatsache nicht verschließen, daß es nicht möglich ist, in der reifen Spore ein Gebilde nachzuweisen, das man für den Kern halten könnte. Die Spore erscheint als ein Protoplasma, das allen Theorien zum Trotze ein, wenn auch minimales, so doch ganz unzweifelhaftes Leben ohne jeden Kern zu führen imstande ist und zwar eine Zeit hindurch, welche der Lebenslänge eines vegetativen, nach jenen Autoren mit einem Kerne versehenen, Individuums gegenuiber, als in Potentia unendlich länger bezeichnet werden muß.

Die Anhänger der Zelltheorie der Bakterien sind mit Hinblick auf die Tatsache, daß die Spore zuerst einen Kern besitzt, ihn dann nicht mehr besitzt, während er in der keimenden Spore wieder auftaucht, - sofern sie sich nicht gewaltsamen Deutungen zuwenden wollen - zu der Anerkennung gezwungen, daß in diesem Falle der Kern während des Lebens schwindet und später wieder auftaucht, eine Tatsache, die, obwohl unter bestimmten Bedingungen nachweisbar, fur andre Zellen bestritten worden ist.

Für mich stehen die Dinge freilich anders. Da infolge meines Beweises der Kernnatur der Strukturkomponenten des Milzbrandbacteriums von einem Schwunde und Auftauchen eines Kerns keine Rede sein kann, so ist es klar, daß es sich nur um einen Ausdruck des morphologischen Metabolismus des Protoplasmas, bestimmter ausgedrickt, um einen Fall von Umwandlung des Chromatins in eine chemisch unterschiedene und gleichzeitig morphologisch anders geartete Substanz handeln könne. Aus meinen oben gemachten Äußerungen geht hervor, daß diese Substanz das Linin ist.

Durch Verwendung der oben dargelegten Doppelfärbung mit Fuchsin und Indophenolblau kann gezeigt werden, daß der Sporenbildung ein Anhäufen von Chromatinkörnchen auf dem fertilen Pole, eine Absonderung derselben vom übrigen Körper vermittels einer Zwischenwand und ein Zusammenfließen der Körnchen vorangeht, worauf die Morpholyse des Chromatins folgt, verbunden mit einer 
chemischen Umwandlung im Sinne der Zuneigung zu den Lininreaktionen. Aus der unfürbbaren Lininspore sehen wir dann bei der Keimung wiederum das Chromatin entstehen. Es ist klar, daß es sich hierbei um ein Wachstum des Chromatins aus den ultramikroskopischen Dimensionen eines Molekülkomplexes über die Grenze des Auflösungsvermögens des Mikroskopes hinaus, so daß es in Form eines sofort mit dem Fuchsin färbbaren Körnchens sichtbar wird, und in der Folge um eine weitere Vermehrung desselben handelt, bis das aus der achromatischen Lininspore entstandene Stäbchen zum größten Teil wieder aus Chromatin besteht.

Besser als andre Beispiele eignet sich gewiß das soeben angeführte, um einesteils den Umstand zu illustrieren, daß der morphologische Metabolismus unzertrennlich mit dem chemischen verbunden ist, anderseits um wahrscheinlich zu machen, daß die Umwandlung. des Chromatins zum Linin eine weitverbreitete Erscheinung, wenn nicht sogar eine allgemein gültige Regel ist. Der Beweis für die letztere Annahme steht freilich noch aus.

Behauptet kann aber werden, daß es keinen bewiesenen Fall von morphologischem Metabolismus gebe, der sich nicht zugleich durch eine Änderung der chemischen Reaktionen der durch den Metabolismus direkt entstandenen Produkte kundgeben würde. "Die Entstehung des Typischen aus dem Atypischen * (Roux) ist sonach stets mit einer chemischen Änderung verbunden.

Daraus ergibt sich der Schluß, daß die hierbei ins Werk tretenden chemischen Vorgänge in direktem causalen Zusammenhange mit dem morphologischen Metabolismus stehen, oder, daß der morphologische Metabolismus ein auf chemischen Vorgängen beruhendes Geschehen sei. Die letzteren sind die Ursache der Morpholyse und der Morphogenese und sie sind daher jene "Fernkräfte ", welche Driesch als die letzte Ursache morphogenetischer Vorgänge in der Ontogenese bezeichnet hat, ohne sie näher zu determinieren.

Die letzte Ursache des morphologischen Metabolismus sind daher die Stoffwechselvorgänge im Protoplasma und dieser Umstand ist zugleich der Schlußstein des Beweises vom elementaren Charakter des oft genannten Geschehens.

\section{SchluB.}

Wie aus dem bisher Angeführten hervorgeht, kann der morphologische Metabolismus auf chemisch-physikalische Vorgänge zurlickgefuhtrt werden, welche auf Grund rou Aggregation kleinster 'Teilchen 
aus einem einphasigen Mutterboden die Morphogenese, auf Grund von Disgregation derselben die Morpholyse - im ersten Falle also die Entstehung eines zweiphasigen Systems, im zweiten aber die Ruickverwandlung eines solchen in ein einphasiges System bewirken.

Mit dieser Vorstellung stimmt völlig überein, was ich über das Verhalten der vital gefärbten Teile bei der Morpholyse und Morphogenese (in den Abschnitten 1 und 5) mitgeteilt habe.

Dieses Verhalten deutet an, daß die Vollendung der Aggregation der kleinsten Bestandteilchen auf Grund chemischer Prozesse identisch ist mit der Vollendung der Morphogenese, d. h. mit der Entstehung der Differenzierung; und umgekehrt, daß die Vollendung der Disgregation der kleinsten Bestandteilchen auf Grund chemischer Prozesse identisch ist mit der Vollendung der Morpholyse, d.h. mit dem Schwunde der Differenzierung.

Daß dem so ist, zeigen auch die Ergebnisse der Merotomie; wie sollte sonst erklärt werden, daß von einer Zelle, aus welcher der Kern operativ entfernt worden ist, schließlich sowohl dieser Kern, als auch das ibriggebliebene Cytoplasma untergehen, ohne die entfernten Teile zu restituieren, wenn die Ausbildung des Kernes nicht auch zugleich die vollendete Aggregation von Bestandteilen bestimmter Qualität wïre? (Siehe auch Abschnitt 8.) Sobald alle diese homologen, in dem völlig ausgebildeten Kern zusammengefaßten Bestandteile aus der Zelle entfernt werden, ist das restierende Protoplasma nicht mehr fähig dieselben auszubilden und beide Komponenten verenden, weil offenbar eine Reihe von chemischen Beziehungen, die früher zwischen denselben bestanden haben, in Wegfall gekommen ist.

Es ist freilich klar, daß der Vorgang der Aggregation und Disgregation der kleinsten Teilchen in keiner Weise mit der Bildung und Auflösung eines Niederschlages (Frscrer) identisch sein kann; trotzdem entzieht sich derselbe keineswegs dem durch die neueren Ergebnisse der physiko-chemischen Forschungen über die Colloide gebildeten Vorstellungskreise. Ich mache diesbezüglich auf die Arbeit von Pictox und Lisder ${ }^{1}$ ) aufmerksam. Nach den Angaben dieser Forscher bleibt ein elektrolytisches Salz, selbst wenn seine Konzentration nicht geniigt, um ein Hydrosol in dessen Gel umzubilden, doch nicht ohne Wirkung, sondern verursacht eine Vergrößerung der das Hydrosol bildenden ultramikroskopischen Teilehen durch Agglu-

1: Plctun and Lixuer, Journ, of the Chem, 61. 1892. 
tination, wodurch das Hydrosol andre physikalische Eigenschaften erwirbt und zugleich Strukturenbildung aufweist.

Wie Strukturen durch Aggregation aus ultramikroskopischen Teilchen entstehen, hat an nicht lebenden Colloiden HARDY $\left.{ }^{1}\right)$ zu zeigen vermocht und es erübrigt mir bloß, auf die auffallende äußere $\ddot{\text { Ahn}}$ lichkeit der von ihm beobachteten Erscheinungen mit denjenigen, welche ich (Abschnitt 1) an lebenden Objekten zu konstatieren Gelegenheit hatte, hinzuweisen.

Als alleinige logische Schlußfolgerung ergibt sich aus diesen Beobachtungsreihen direkt, daß die Zweiphasigkeit des Protoplasma keine wesentliche Eigenschaft desselben ist, sondern da $B$ - wie die Erscheinungen des morphologischen Metabolismus an den Strukturen des Cyto- und Caryoplasma lehren -- auch die "flussige "Phase desselben, von welcher Heitziraxn behauptet hatte, daß sie nicht lebt, notwendigerweise lebende Substanz enthalten muß. Sie muß dieselbe ebenso enthalten, wie die Intercellularsubstanzen, woran man früher anch gezweifelt hat, ja was selbst bis beute noch nicht allgemein durchzudringen vermochte, wiewohl dies durch exakte Beobachtungen bekräftigt worden ist. (Siehe die Arbeiten der Grawitzschen Schule über die histologischen Vorgiinge bei der Entzündung der verschiedensten Gewebe.)

Denn wäre dem nicht so, so erschiene der morphologische Metabolismus als ein der Generatio aequivoca gleiches Geschehen, was allen Tatsachen der jetzigen Wissenschaft widerstreiten würde. Erkennt man jedoch an, daß auch die flüssige Phase des Protoplasmas einen Teil der lebenden Substanz enthält, so wird man sich umgekehrt mit allen physikaliseh-mechanischen Tatsachen, mit welchen uns das Studium der Colloide bereichert hat, in Übereinstimmung befinden.

Diese Übereinstimmung ist freilich als keine essentielle aufzufassen.

Da meinen Ausfuhrungen zufolge die mikroskopische homogene, "flüssige Phase des Protoplasmas auch einen Teil der lebenden Sub$\operatorname{stanz}$ entbalten muß, so ist es fernerhin klar, daß die eigentliche Protoplasmastruktur auf ultramikroskopischem Gebiete zu suchen ist; dies hat jedoch keine andre Bedeutung als die, daß das lebende Protoplasma urspringlich mikroskopisch strukturlos ist, d. h. in physikalisch-chemischem Sinne ein einphasiges System bildet.

1. Hardy, Journ. of' Physiology. 24. 1899. 
Es ist derselbe Schluß, zu welchem Hardy auf Grund andrer Erwägungen gelangt ist.

Daß dieser Umstand fü die Entwicklung der biochemischen Prozesse nicht hinderlich zu sein braucht, ist von Pauli ${ }^{1}$ ) gezeigt worden.

Dem ersten Anscheine nach schiene es, daß der morphologische Metabolismus des Kerns in einem krassen Widerspruche zu den in den Lebensbezeugungen des Chromatins entdeckten Gesetzmäßigkeiten stehe, welche in Boveris Individualitätstheorie zusammengefaßt erscheinen.

Auf Grund der Arbeiten von Ral3L, deren Resultate auf viele Zellen ausgedehnt worden sind, erkennt man bekanntlich eine bestimmte Struktur des Chromatins als gesetzmäiBig an und auf Grund dieses gesetzmäBigen Verhaltens wurden weitreichende biologische Theorien konstruiert. Nach RABL muß selbst in jedem ruhenden Kerne eine bestimmte polare Anordnung der Chromatinschleifen bestehen, welche durch die Konvergenz derselben zur Sphäre charakterisiert wird. Für diese, ans dem Verhalten des Chromatins im Beginne der Mitose abgeleitete Voraussetzung hat besonders Boveri eine große Anzahl von Belegen beigebracht.

Die größte Mehrzahl der Kerne bietet jedoch kein genügend uberzeugendes Material für jene Behauptung. Es ist keineswegs bewiesen, daß das in bestimmten Zeiten in den Kernen zu beobachtende Netzwerk auch tatsächlich dem Zustande der Kernruhe entspricht. Ich bringe das von mir über diese Angelegenheit im Abschnitte 2 Angefuihrte in Erinnerung.

Es besteht daher keine Notwendigkeit, welche nach sich zöge, den morphologischen Metabolismus mit den Tatsachen der Individualitätstheorie in Gegensatz zu bringen.

Das ergibt sich aus gewissen Beobachtungen Tellyesniczkrs.

Bei der Differenzierung des Kernfadens aus dem diffusen $*$ Kernsafte durch Aggregation feinster ursprünglicher Formationen geben nämlich diese letzteren im Anfange durchaus keine Polarität zu erkennen; dieselbe tritt erst später auf, nachdem der Faden bereits bedeutend an Dicke zugenommen hatte.

Desgleichen bleibt bei dem Untergange der Tochterchromosomen

1) Pauli, PfuÜgirs Arch. 67, 71, 78. - Pauli und Rona, Hofmeisters Beitr. 2. 1902 . 
die Polarität der Schleifen noch lange ausgedritickt, schwindet zuletzt aber doch.

Auch K. Bonnevie ${ }^{1)}$ gibt an, daß die Schleifen in den Ovogonien von Enteroxenos östergreni manchmal überhaupt keine polare Anordnung anfweisen; in andern Fällen ist dieselbe zwar vorhanden, jedoch nur schwach ansgesprochen.

Derartige Beobachtungen bilden gewiß die Briicke, welche das Gesetz des morphologischen Metabolismus des Protoplasmas mit der Individualitätstheorie verbindet.

Zum Schlusse seien mir einige Worte von ganz allgemeinem Inhalte gestattet.

Im Abschnitte 10 wurde unter Benutzung des Gedankenganges von DRIESCH gezeigt, daß der morphologische Metabolismus als elementarstes morphogenes Geschehen insofern ein Problem darbietet, als es sich um die Lokalisation dieses Geschehens im Protoplasma handelt.

Im Abschnitte 9 wurde der Nachweis geliefert, daß der morphologische Metabolismus nur unter der Voraussetzung möglich ist, daß das Protoplasma ein harmonisch-äquipotentielles System darstellt.

Ohne weiteres ist es weiterhin klar, daß der morphologische Metabolismus ein von der Einwirkung äußerer Reize unabhängiges Geschehen sei.

Ich habe bereits früher daran erinnert, daß die Lokalisation einer ohue Einfluß äuBerer Reize in einem harmonisch-äquipotentiellen Systeme vor sich gehenden Differenzierung Driesch als ein Problem sui generis, als ein besonderes, aus den Gesetzmäßigkeiten der anorganischen Disziplinen unerklärbares Geschehen erscheint.

Durch die Ausfibhrungen des Abschnittes 11 konnte ich jedoch beweisen, daß die Ursachen des morphologisehen Metabolismus chemische Vorg:inge sind, was auch experimentell bewiesen werdeu kann. Durch chemische Änderung des Nährbodens kann erzielt werden, daß das Anthraxbacterium keine Sporen herausbildet. Durch Änderung der Ernährung des Seeigeleies vermittels Einbringens in Lösungen bestimmter chemischer Stoffe kann die Ausbildung von Centrosomen erzielt werden und zwar je nachdem man verschiedene Agentien an-

1 Boxnevis, D. Verh. des Chromatins in den Keimzellen von Enteroxeros östergreni. Anat. Anz. 1905. 
wendet, ans verschiedenen Teilen des Protoplasmas. Dieser Umstand kann selbstverstïndlich nicht so gedeutet werden, als wenn dies der Behauptung von der Unabhängigkeit des Metabolismus von äußeren Reizen widersprechen würde.

Meiner Meinung nach könnte also wolll von einem Probleme sui generis gesprochen werden, wenn die Lokalisation des morphologischen Metabolismus konstant, stets dieselbe wäre unter allen Umständen; dann läge ein Geschehen vor, das aus den Gesetzmäßigkeiten der anorganischen Disziplinen unerklärlich, vitalistisch im Sinne von Driesch wäre.

Doch wird die hier gemachte Voraussetzung durch die Tatsachen nicht bestätigt. Man kann direkt sehen, daß nach der Morpholyse sich neue Kerne an beliebiger Stelle, in beliebiger Form und Anzahl bilden können; bei demselben Individuum können unter verschiedenen äußeren Bedingungen (also durch Einwirkung verschiedener chemischer Einflitsse) die Differenzierungen an verschiedenen Orten entstehen (die Centrosomen im Kerne oder im Cytoplasma, oder ans beiden, die Spindel gleichfalls usw.); die Sporen der Bakterien können an verschiedenen Stellen derselben zur Entstehung gelangen.

Kurz, man sieht, daß die Lokalisation des morphologisehen Metabolismus im allgemeinen Sinne beliebig, variabel ist. Es ist offenbar, daß sich der Zweck der Morphogenese und Morpholyse nicht durch die Lokalisation, sondern nur durch die Funktion kundgibt, welch' letztere jedoch von dem Stoffwechsel, d. h. von chemischen Vorgängen, untrennbar ist.

Chemische Vorgänge, welche - wie oben dargelegt wurde die Ursache des morphologischen Metabolismus sind, hat jedoch DRIESCH selbst nach dem Vorbilde von Schopenhader als solche anerkannt, bei welchen die Ursache dem Effekte entspricht und zwar in dem Sinne, daß die erstere in dem letzteren in irgendwelcher Form (mit einigen Charakteren) wieder auftritt. Dieser Forderung entspricht der morphologische Metabolismus in weitgehendstem Maße, denn die morphologischen Umwandlungen sind, wie ich im Abschnitte 11 gezeigt habe, stets mit chemischen verbunden.

Ein Geschehen aber, dessen Ursache dem Effekte entspricht, ist nach Driesch niemals ein vitalistisches Geschehen.

Diese Schlußfolgerung gilt nach dem Angeführten natuirlich auch für den morphologischen Metabolismus und zwar selbst dem Umstande zum Trotze, daß dieses Geschehen, wie im Abschnitte 9 des Besonderen bewiesen wurde, ein Antwortgeschehen ist. 
356 Vladislav Rúžička. Der morphologische Metabolismus usw.

Zum Schluß hebe ich ausdrücklich hervor, daß die Resultate der von mir in vorliegender Arbeit unternommenen Analyse keineswegs generalisiert, sondern nur auf jene Fälle bezogen werden sollen, in welchen die Prämissen derselben, $d . h$. die Tatsachen, welche den in den Abschnitten 1-6 angefuhrten analog sind, zutage treten.

Prag, im Dezember 1905. 